\title{
Progressive Failure Analysis on Textile Composites
}

\author{
Dianyun Zhang* and Anthony M. Waas ${ }^{\dagger}$ \\ Department of Aerospace Engineering, University of Michigan, Ann Arbor, MI 48109, USA \\ Chian-Fong Yen ${ }^{\ddagger}$ \\ ARMY Research Laboratory, Aberdeen Proving Ground, MD 21005, USA
}

The flexrual response of a layer-to-layer orthogonal interlocked 3D textile composite (3DTC) has been investigated through quasi-static three-point bending. Fiber tow kinking on the compressive side of the flexed specimens has been found to be a strength limiting mechanism for both warp and weft panels. The digital image correlation technique has been utilized to map the deformation and identify matrix micro-cracking on the tensile side prior to the peak load in the warp direction loaded panels. It is shown that the geometrical characteristics of textile reinforcement play a key role on the mechanical response of this class of material. A mechanism based multiscale computational model that considers the influence of textile architecture has been developed to capture the observed damage and failure characteristics. The fiber tows and surrounding polymer matrix are modeled at the mesoscale, while the 3DTC is homogenized at the macroscale. The pre-peak nonlinear response of the fiber tow is modeled using a novel, two-scale model, in which the subscale micromechanical analysis is carried out in close form. The influence of the matrix microdamage at the microscale manifests as the progressive degradation of the fiber tow stiffness at the mesoscale. The post-peak strain softening responses of the fiber tows and the surrounding polymer matrix are modeled through the smeared crack approach (SCA). The load-deflection response, along with the observed damage events, including matrix cracking, tow kinking, and tow tensile breakage, are successfully captured through the proposed computational model. Therefore, the proposed multiscale model is suitable for progressive damage and failure analysis of 3DTCs and specifically to study the influence of textile architecture on macroscopic response.

\section{Nomenclature}

$E_{e} \quad$ Elastic Young's modulus

$E_{s} \quad$ Secant Young's modulus

$E_{1} \quad$ Axial Young's modulus

$E_{2} \quad$ Transverse Young's modulus

$\nu_{e} \quad$ Elastic Poisson's ratio

$\nu_{s} \quad$ Secant Poisson's ratio

$\nu_{12} \quad$ Axial Poisson's ratio

$\nu_{23}$ Transverse Poisson's ratio

$G_{12}$ Axial shear modulus

$G_{23}$ Transverse shear modulus

$K_{23}$ Plane-strain bulk modulus

$\boldsymbol{\sigma}$ Stress

$\sigma_{e q} \quad$ Equivalent stress

$\sigma_{y} \quad$ Yield stress

*PhD Candidate, Department of Aerospace Engineering, University of Michigan, 1320 Beal Avenue, Ann Arbor, MI 48109.

${ }^{\dagger}$ Felix Pawlowski Collegiate Professor of Aerospace Engineering, Department of Aerospace Engineering, University of Michigan, 1320 Beal Avenue, Ann Arbor, MI 48109, Fellow AIAA.

${ }^{\ddagger}$ Army Research Laboratory, Aberdeen Proving Ground, MD 21005. 


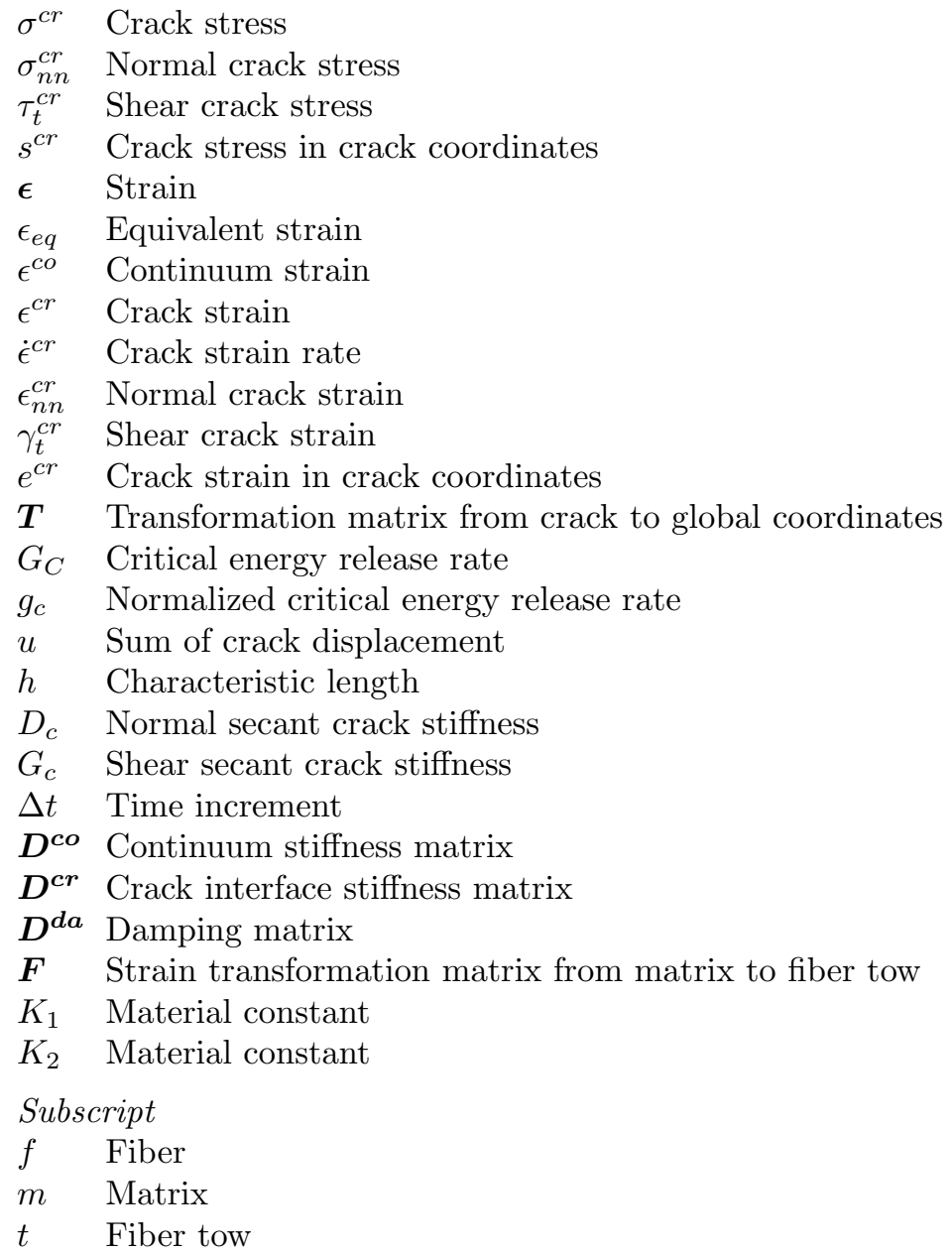

\section{Introduction}

3D textile composites (3DTCs) are attractive structural materials for a variety of reasons. They are increasingly used in several aerospace, civil, mechanical and naval industrial applications. The term textile here is referred to an interlaced structure, known as a dry preform, which consists of fiber tows that are either woven or braided together. Compared with pre-preg laminated composites, 3DTCs offer better resistance to delamination while demonstrating a cost advantage. It has been shown in previous studies that 3D textile composites possess enhanced mechanical performance and the ability to contain (localize) the area of damage due to delamination. ${ }^{1,2,3}$

In order to obtain a thorough understanding on the deformation response of textile composites, a number of analytical models have been developed to compute the elastic properties, including in-plane moduli ${ }^{4,5}$ and bending stiffness. ${ }^{6}$ It has been pointed by many researchers ${ }^{7,8,9}$ that although geometric distortions resulting from curing and consolidation have little effect on elastic moduli, these manufacturing induced imperfections can greatly affect the damage behavior and the resulting mechanical properties such as strength, strain to failure, and fatigue life. The importance of incorporating any unintended geometric deviations of textile architecture into micromechanics based finite element modeling has been addressed recently by Zhang et al. ${ }^{10}$

The flexural response of textile composites has been investigated by Cox et al. ${ }^{11}$ and Quek et al. ${ }^{12}$ In their studies, fiber tow kinking on the compressive side of the specimen was reported, and this type of failure mode was identified as a strength limiting mechanism. In the literature, the compressive strength of a unidirectional composite has been studied by Budiansky and Fleck, ${ }^{13}$ Kyriakides et al.,${ }^{14}$ Lee and Waas, ${ }^{15}$ Feld et al. ${ }^{16}$ The main physical event associated with kink band formation is the rotation of the fibers in a band within a locally degrading matrix, with or without splitting. The rotation of fibers gives rise 
to high localized shear strains and transverse strain that drive further degradation of the matrix material between the fibers within a tow. The shear degradation in turn increases the rotation of the fibers creating a positive feedback loop that culminates in a limit-load type instability. During the kink band formation, the degrading matrix can also split (transverse tensile failure), leading to a competition between splitting and intact kinking, as recently studied by Prabhakar and Waas, ${ }^{17,18}$ following earlier work by Lee and Waas. ${ }^{15}$

The focus of this paper is to investigate the failure mechanisms in the flexural response of a quasi-statically loaded 3DTC panel through experiment and an accompanying mechanism based model that considers the influence of textile architecture. The deformation response is analyzed through a multiscale computational model, in which the fiber tows and surrounding polymer matrix are modeled at the mesoscale, while the 3DTC is homogenized at the macroscale. The pre-peak nonlinear response of the fiber tow is modeled using a novel, two-scale model, in which the subscale micromechanical analysis is carried out in close form. The post-peak strain softening responses of the fiber tows and the surrounding polymer matrix are modeled through the smeared crack approach (SCA). The predictive capability of the proposed model is illustrated by comparing the computed results with the experimental investigation including the load-deflection response and the failure characteristics, such as matrix cracking, tow kinking, and tensile fracture of the tows.

\section{Material System}

The material investigated in this paper is a layer-to-layer orthogonal interlocked 3DTC, in which the warp tows are woven through five weft fiber layers alternating with a period of four along the weft direction to achieve a repeated textile pattern, as schematically shown in Figure 1. The dry textile preform is made of S2 glass fibers that are provided by Albany Engineered Composites, Inc., NY. Impregnation and cure of the textile preform were carried out at the Army Research Laboratory in Aberdeen, MD, using a vacuum assisted resin transfer molding (VARTM) process. VARTM is adapted from traditional Resin Transfer Molding (RTM) by replacing the upper half mold with a vacuum bag to enhance the impregnation of the fiber reinforcements. Details of the VARTM technique and fabrication process are provided in Refs. ${ }^{19,20}$ The matrix is SC-15 epoxy resin with two-phased toughened thermoset polymer containing part A (resin mixture of diglycidylether epoxy toughener) and part B (hardener mixture of cycloaliphaic amine poluoxylalkylamine). ${ }^{21}$ The elastic properties for the fiber and matrix are summarized in Table 1.

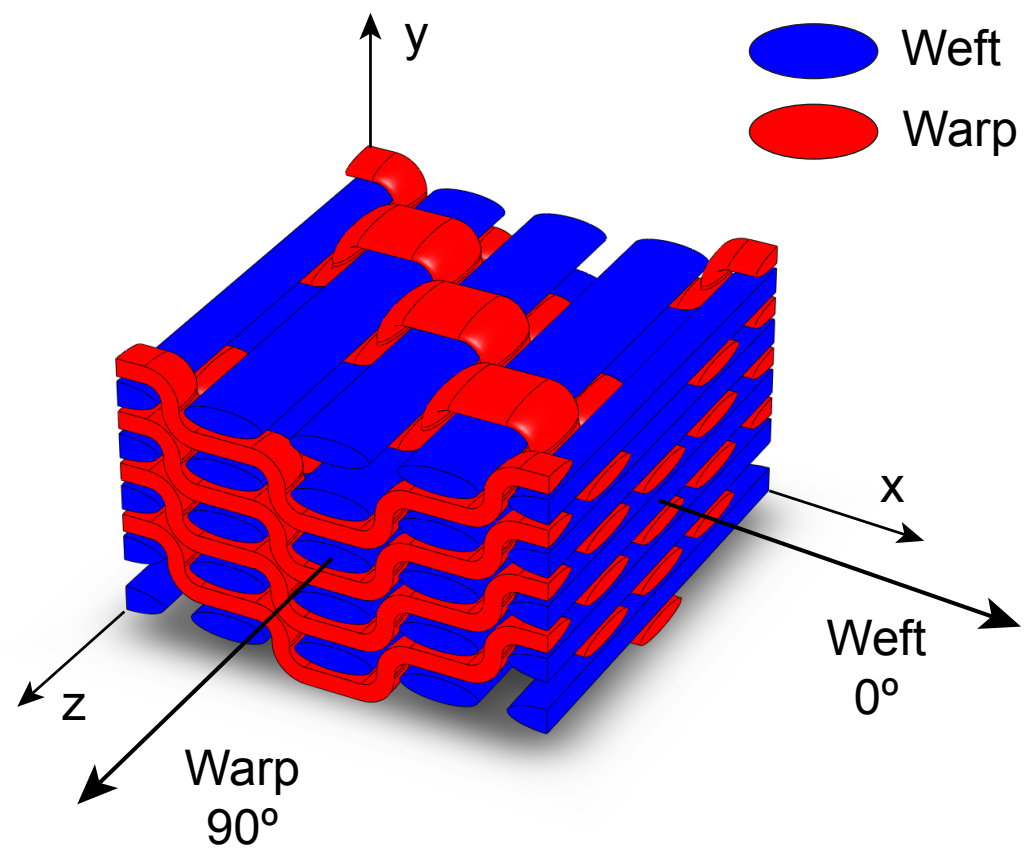

Figure 1. Ideal textile architecture of the layer-to-layer interlock glass fiber 3DTC.

The layer-to-layer architecture is vulnerable to the mold pressure during the fabrication process due to 
Table 1. Elastic properties for S-2 glass and SC-15 matrix.

\begin{tabular}{ccc}
\hline & $E(\mathrm{GPa})$ & $\nu$ \\
\hline S-2 Glass Fiber & 93.8 & 0.23 \\
\hline SC-15 Matrix & 2.49 & 0.35 \\
\hline
\end{tabular}

the large undulation of the warp weavers, as schematically shown in Figure 2. In VARTM process with single-sided mold, atmospheric pressure is exerted on the textile preform through the vacuum bag covering. The applied pressure on the preform forces the fiber tows to be settled in a new position that is different from the ideal designed one. The cross-sectional microscopic images, shown in Figure 3, clearly shows this manufacturing induced geometric imperfection in the final textile composite panels. It has been pointed out by Song et al. ${ }^{22}$ that each manufacturing process is associated with a unique set of characteristics that result in a produced part deviating from the expected ideal geometry. The set of such deviations, which is unique to each manufacturing process, is termed the "manufacturing imperfection signature". Obtaining the manufacturing imperfection signature of the textile composite is important to determine the damage characteristics, such as strength, strain to failure, and fatigue life. ${ }^{7,8,9}$
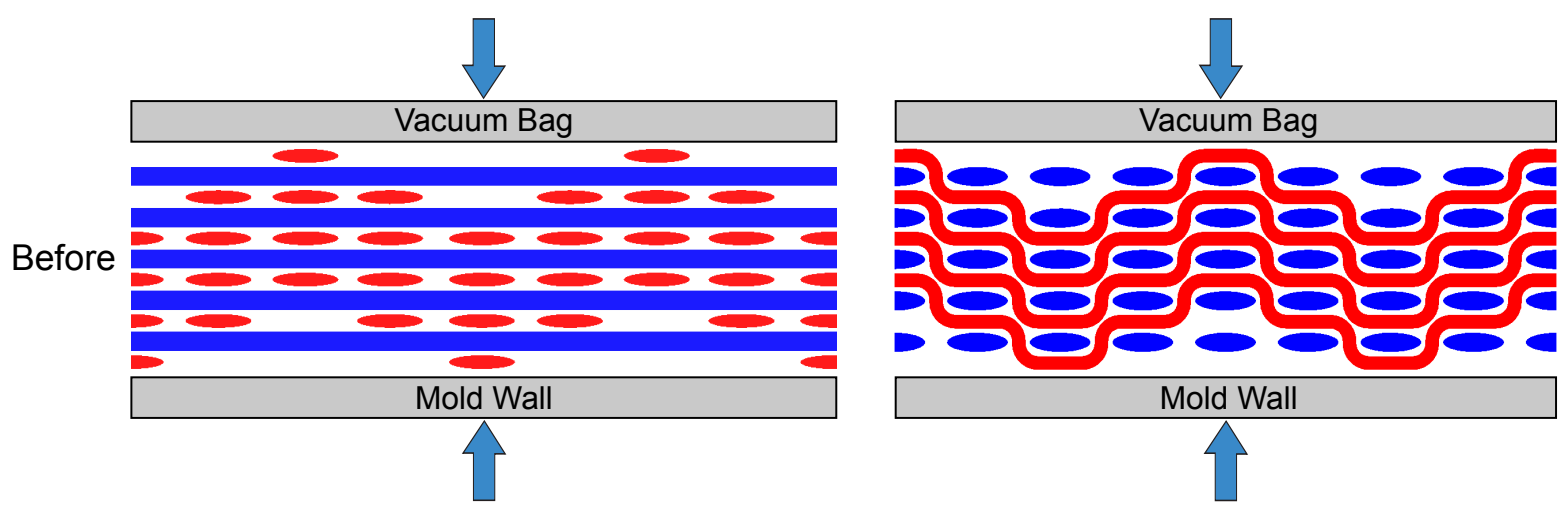

(a) Pressure is exerted during the curing cycle.
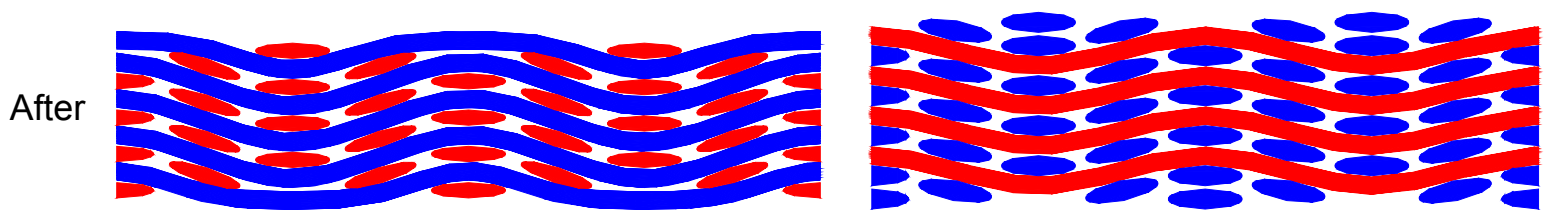

(b) Schematics of the actual textile architectures.

Figure 2. Manufacturing effect on the textile architectures.

Measurements were taken on the cross-sectional micrographs shown in Figure 3 to characterize the geometry of the textile architecture. Fiber tow is idealized to undulate as a sinusoidal path with an elliptical cross section. The measured tow dimensions are summarized in Table 2. The fiber volume fraction within a fiber tow is determined to be $60 \%$ by analyzing a scanning electron microscopic (SEM) image. The volume fraction of warp, weft, and matrix provided by the manufacturer are $24.78 \%, 32.76 \%$, and $42.45 \%$, respectively. ${ }^{10}$

\section{Experimental Results}

Three-point bend tests were performed using a screw-driven Instron machine at a loading rate of 1 $\mathrm{mm} / \mathrm{min}$ to achieve a quasi-static loading condition. The center load and load point displacement were 


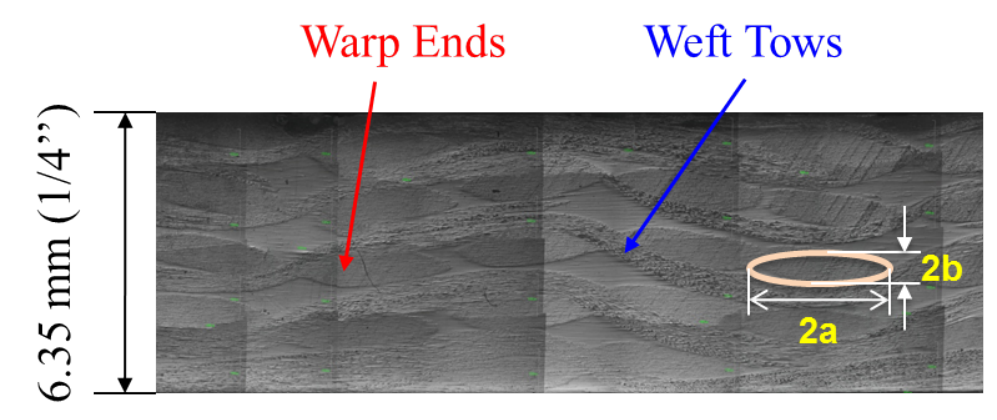

(a) Weft cross section.

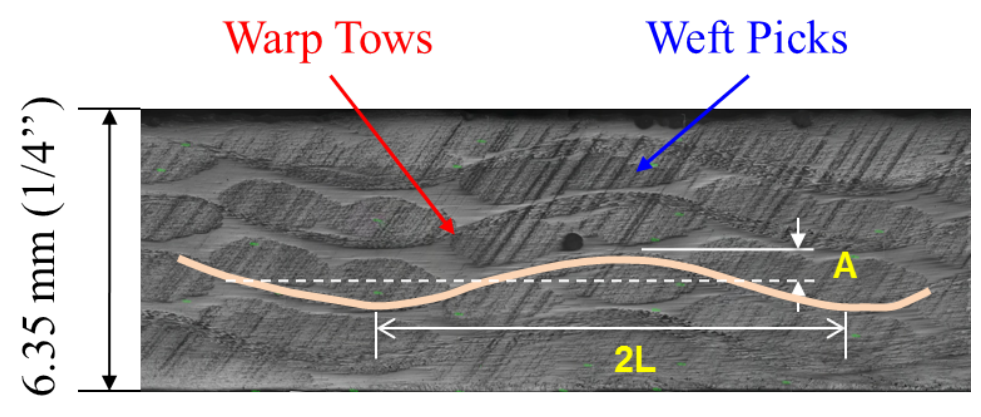

(b) Warp cross section.

Figure 3. Optical micrographs. The fiber tow is idealized to undulate as a sinusoidal wave with an elliptical cross section.

Table 2. Fiber tow geometry.

\begin{tabular}{ccc}
\hline & Weft tow & Warp tow \\
\hline Major axis (2a) (mm) & $2.15 \pm 0.11$ & $2.51 \pm 0.13$ \\
Minor axis (2b) (mm) & $0.62 \pm 0.05$ & $0.53 \pm 0.05$ \\
Wave length (2L) (mm) & $11.80 \pm 0.15$ & $9.29 \pm 0.16$ \\
Amplitude (A) (mm) & $0.74 \pm 0.07$ & $0.45 \pm 0.03$ \\
\hline
\end{tabular}

recorded, as schematically shown in Figure 4. Specimens were cut along the two major fiber directions, warp and weft, to study the architecture-dependent effect. The specimen dimensions are as follows: $120 \mathrm{~mm}$ long, $6.23 \mathrm{~mm}$ thick, and $19 \mathrm{~mm}$ wide, with a bottom span of $90 \mathrm{~mm}$. In order to investigate the failure modes associated with the deformation histories, the outer surface of the specimen is speckled, which can be subsequently used to obtain surface strain fields via a digital image correlation (DIC) technique. Images of the outer surface were taken during the deformation using a 12 Megapixel camera at 5-s time intervals. The full field surface strain histories were obtained via the DIC software ARAMIS.

Representative load-displacement responses are shown in Figure 5(a). The load presented is normalized with respect to the width of the specimen such that the variation in specimen width due to cutting can be accounted for. The weft loading direction shows higher initial stiffness than the warp direction due to the fact that there is one additional axial tow in the weft direction to carry the bending moment. Overall for both directions the woven composites exhibit a similar softening type response, more representative of metals, indicating considerable damage tolerance of this type of material. When the panel was loaded in the weft direction, the first damage occurred at the point that deviates from the proportional loading due to fiber tow kinking on the compression side. The kink band formation can be better observed under a SEM, as shown in Figure 6. The DIC patterns before and after the first damage occurrence are shown in Figure 5 (b). However, when the material was loaded in the warp direction, matrix cracking occurred on the tension 


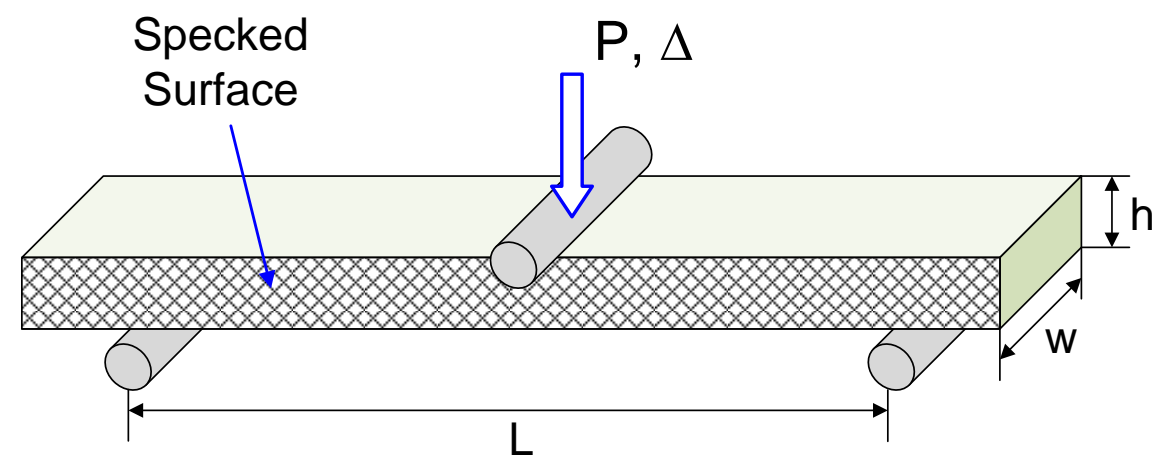

Figure 4. Schematic of three-point bend test configuration. The side surface of the specimen is speckled as indicated.

side and progressed in the center of the specimen prior to the kinking on the compression side. These cracks were captured by the DIC patterns as shown in Figure 5(c). The surface strain contours show the area of strain localization due to the textile architecture, indicating the location where the matrix materials start to crack. In both cases, kink banding signals the onset of significant non-linearity.

It is worth noting that tensile matrix cracking has little impact on the recorded load, whereas the peak load is determined by the fiber tow kinking strength. This agrees with the findings reported in Refs. ${ }^{7,12}$ that kink banding is the strength controlling mechanisms of failure. The observed damage characteristics indicate the importance of the textile architecture on the failure mechanisms of this class of materials. Hence, a textile architecture-based numerical model that incorporates constitutive relations that encompass damage and failure is expected to predict the failure modes observed in the experiment, as discussed in Section IV. 


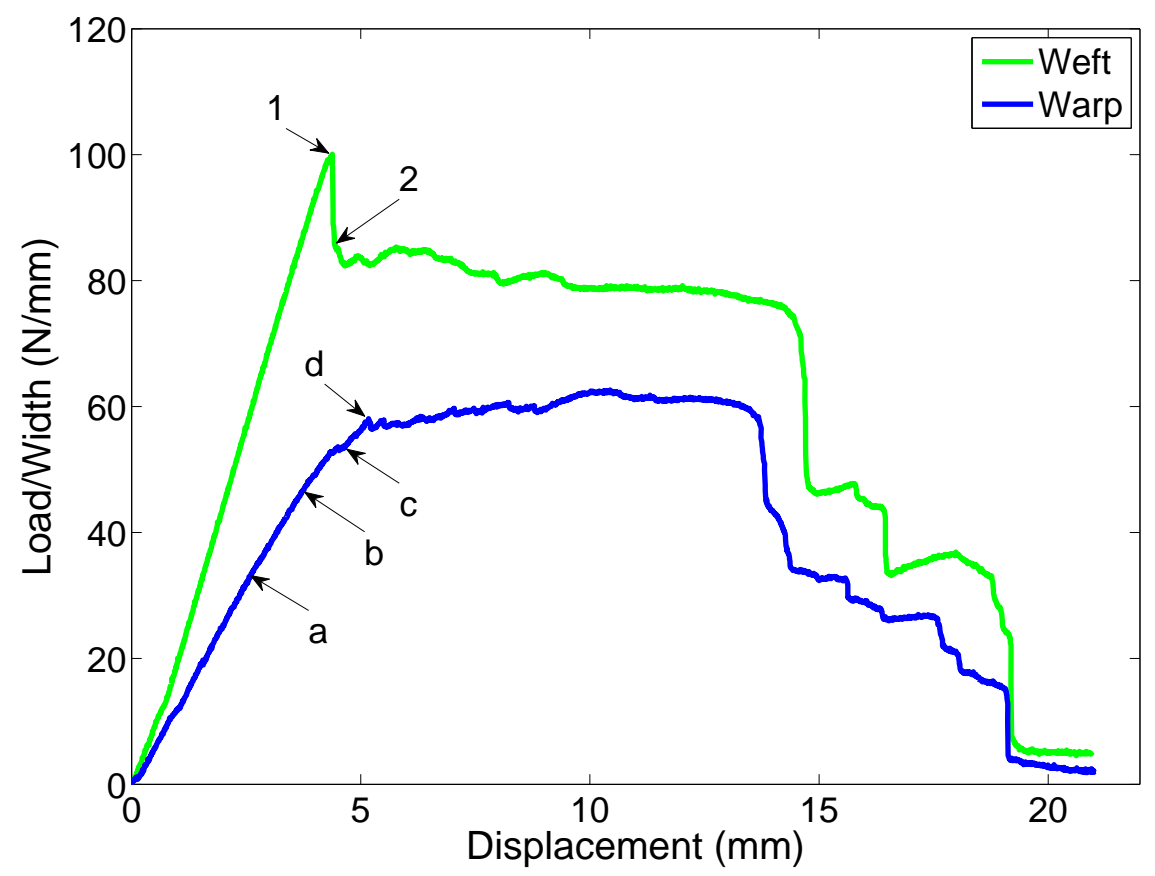

(a) Experimental load-displacement curves

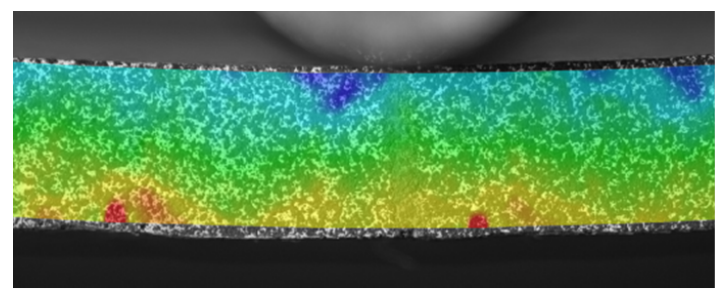

At point (1): before the first damage

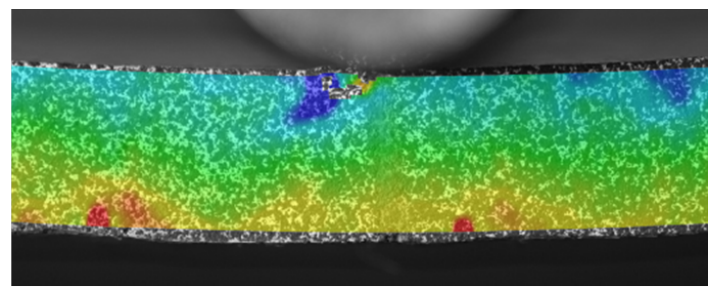

At point (2): after the first damage

(b) Axial surface strain patterns (weft)

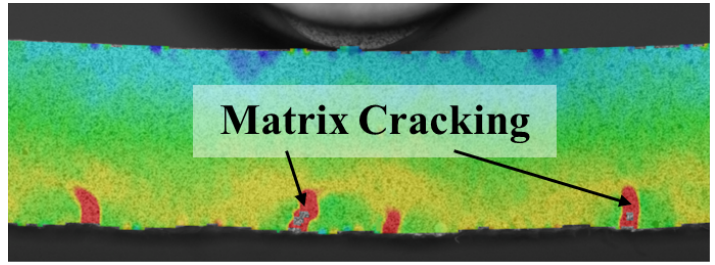

At point (a)

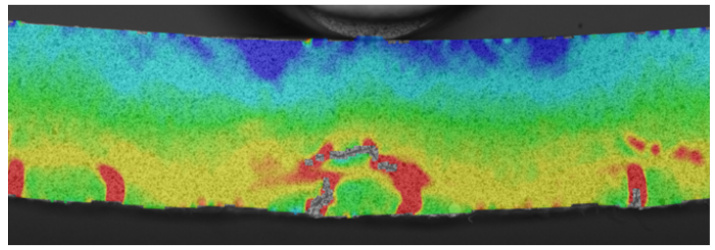

At point (c)

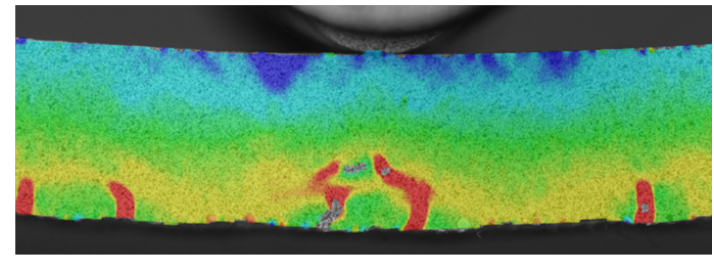

At point (b)

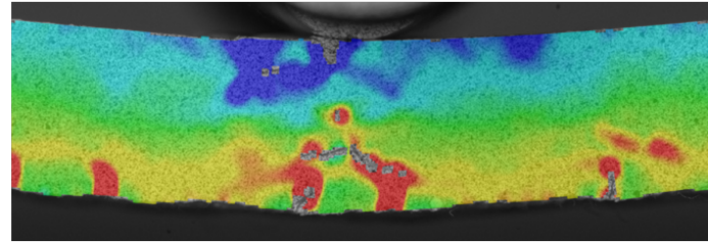

At point (d)

(c) Axial surface strain patterns (warp)

Figure 5. Representative load-displacement curves for the 3DTCs subjected to quasi-static three-point bend tests. Surface strain patterns captured the damage occurrences. 


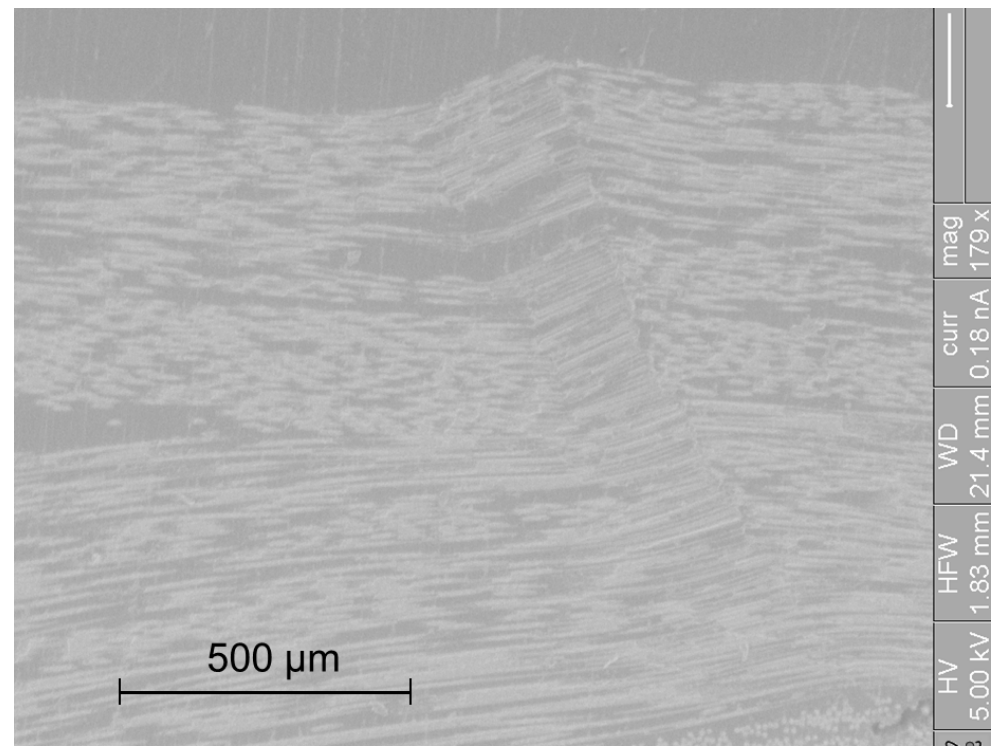

Figure 6. Kink band formation on the compression side of the specimen.

\section{Multiscale Computational Model}

Experimental observations suggest that the numerical model should consider the influence of textile architecture on the macroscale response of 3DTCs. The proposed mechanism based finite element analysis (FEA) model contains three scales as illustrated in Figure 7. For the region that is far away from the loading point (or far away from "hot spots"), the composite beam is modeled as a homogeneous linearelastic orthotropic solid since no damage event is observed. The mesoscale model that contains the details of the textile architecture, including the fiber tows and surrounding polymer matrix, is used in the region in the vicinity of the loading point, where multiple failure modes have been observed. The mesoscale model is a collection of representative unit cells (RUCs) created based on the micrograph in Figure 3 using the measured tow geometry summarized in Table 2. In order to obtain the nonlinear constitutive relation of a single fiber, micromechanical analysis is carried out at the fiber and matrix level, referred to as the microscale. The determination of fiber tow response is discussed in Section VI.

As discussed in Section II, manufacturing induced effects on the textile architecture indicate rotations of tows and interactions between each other. Modeling the full repeated textile pattern requires advanced topology techniques and may result in meshing difficulties. At this point two different mesoscale models are proposed with a focus on cross section. In each model only a unit width of fiber tows were modeled along its axial direction, as illustrated in Figure 8. The tows running along the bending axis (or along the width) are assumed to be straight. 3D linear tetrahedron solid elements are used in the mesoscale model, whereas the homogenous model is meshed using 3D linear brick elements.

The connection between the micro and homogenous model is enforced by requiring displacement continuity between the two regions across the common boundary, as shown in Figure 9. Since only a narrow width of the specimen is modeled, it is assumed that the flexural response is uniform through the width. Symmetric boundary conditions with respect to the z-axis are imposed on the surface EFGH to prevent out-of-plane movement. The top roller is modeled as a rigid body with applied displacement along $y$ - direction. Both the left bottom edge $\mathrm{BF}$ and the right bottom edge $\mathrm{CG}$ are fixed in $y-$ and $z$-directions to achieve a three-point bend loading condition. 


\section{Homogenized Macroscale Model}

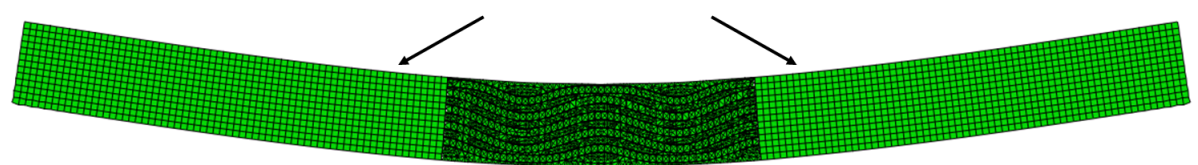

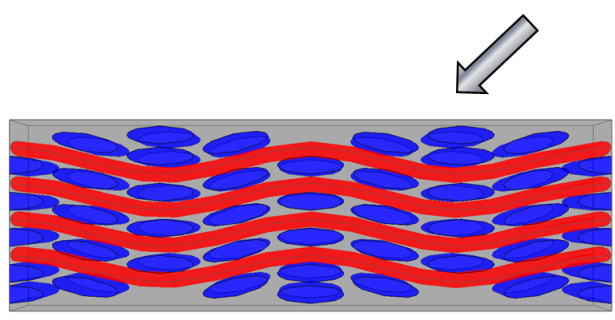

Mesoscale Model

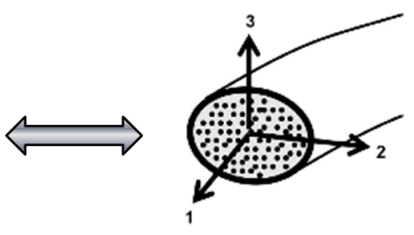

Microscale Model

Figure 7. Illustration of multiscale modeling strategy.

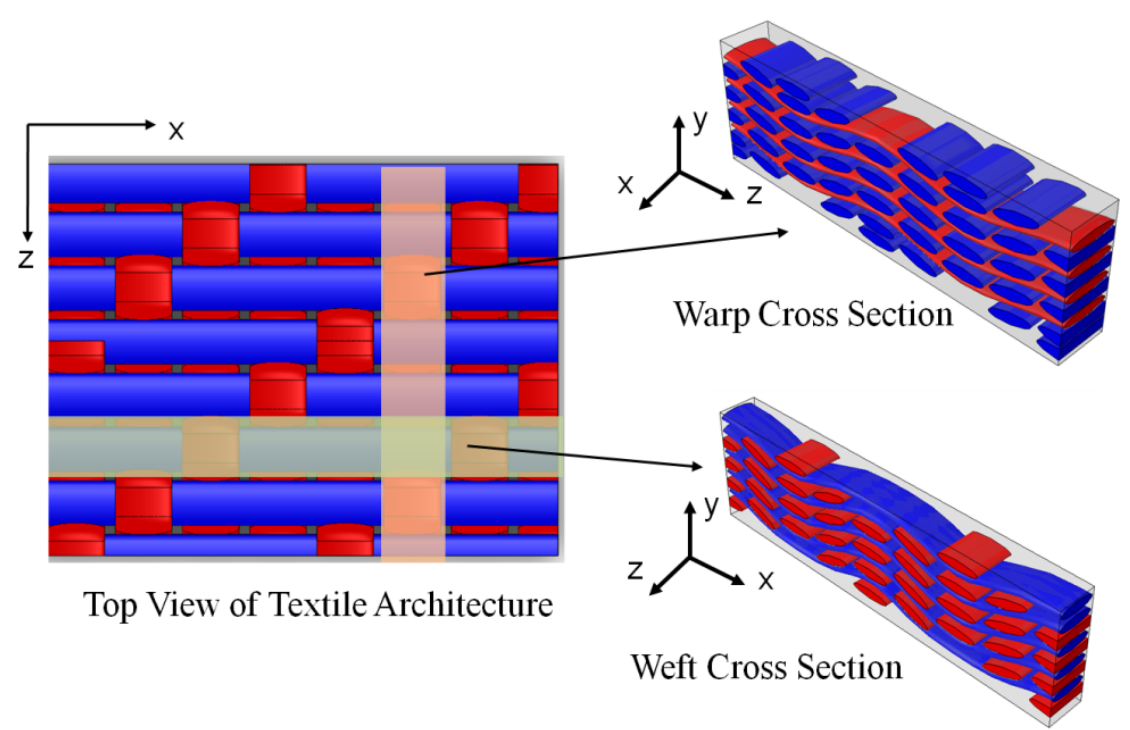

Figure 8. Construction of mesoscale model. In each model, only a unit width of fiber tows are modeled along its axial direction. The tows running along the width are assumed to be straight.

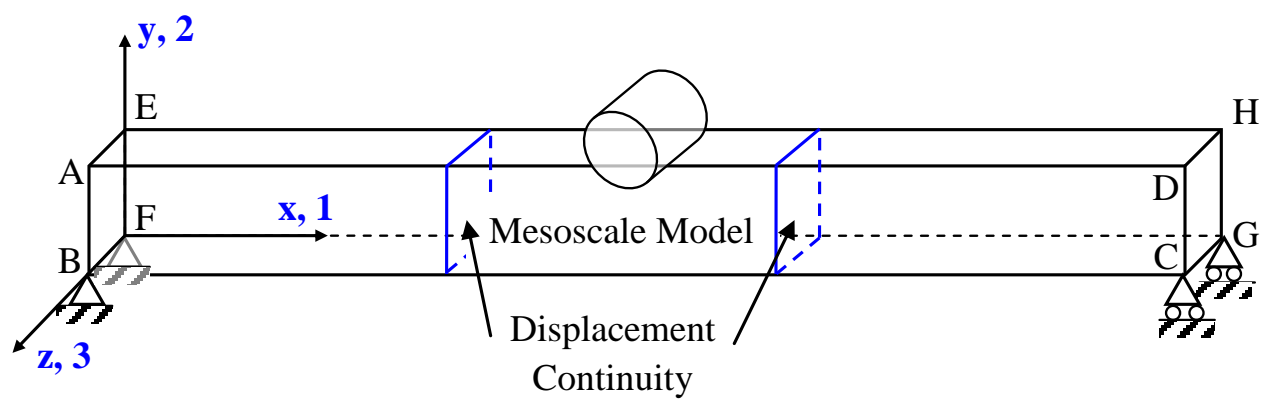

Figure 9. Boundary conditions of the global-local FEA model. 


\section{Matrix Constitutive Model}

Matrix cracking is observed on the tensile side of a flexed 3DTC panel and progressed throughout the specimen. In addition, the polymer matrix material exhibits a nonlinear stress-strain response before failure. In this paper, damage and failure are distinguished in such a manner that damage governs any nonlinear response that preserves the positive definiteness of the material stiffness tensor; whereas failure is defined as the microstructural changes that result in a post-peak strain softening in the stress-strain response. Figure 10 shows a representative full constitutive response of a polymer matrix including damage and failure behavior. The pre-peak nonlinear behavior is attributed to matrix microdamage due to the growing of voids and flaws in the polymer material, and is considered as a damage mechanism. The accumulation of matrix microdamge finally results in matrix macroscopic cracking that is categorized as a failure mechanism due to the loss of positive definiteness of the material tangent stiffness tenor.

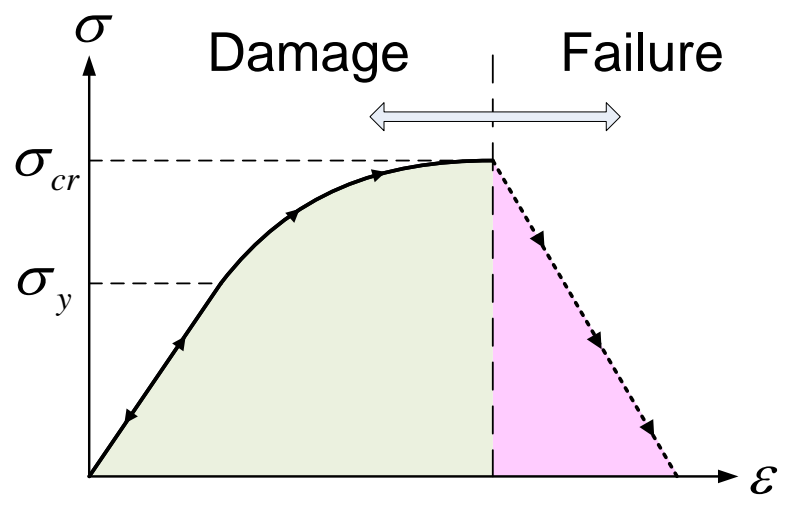

Figure 10. Schematic of matrix constitutive behavior including damage and failure.

In this section, the modeling strategy for an isotropic matrix material is presented, including damage and failure analysis. The matrix microdamage, manifested as pre-peak nonlinearity in the stress-strain response, is modeled using a modified $J_{2}$ deformation theory of plasticity, as formulated in Section V.A. The secant moduli are degraded with the progression of microdamage, however, the positive definiteness of the stiffness tensor is still preserved. When a critical stress state is reached, failure is initiated and the post-peak strain softening behavior is modeled through the SCA to capture matrix macroscopic cracking, as given in Section V.B. The implementation of this matrix material model to the mesoscale textile architecture based FEA model is presented in Section IV.

\section{A. Modeling the Microdamage in Polymer Matrix Based upon Modified $J_{2}$ Deformation Theory of Plasticity}

It has been shown by Sicking ${ }^{23}$ that a polymer matrix exhibits limited loading path independence behavior through combined tension and torsion tests. Hence, for such an isotropic material, the state of stress can be uniquely determined from the state of strain through a secant modulus as long as the material does not experience unloading. It further assumes that the evolution of damage is an irreversible process, therefore, once the matrix stiffness tensor is degraded due to microdamage, it cannot be recovered. Such behavior suggests that a modified $J_{2}$ deformation theory of plasticity be employed to model the nonlinear stressstrain relation of this type of material. In the present modified constitutive model, the degrading secant moduli are utilized to compute the material stiffness tensor during unloading.

In order to utilize a uniaxial stress-strain curve to determine the material response under multi-axial loading, two equivalent variables, the equivalent stress, $\sigma_{e q}^{m}$, and the equivalent strain, $\epsilon_{e q}^{m}$, are introduced and related through a secant Young's modulus, $E_{s}^{m}$, as $\sigma_{e q}^{m}=E_{s}^{m} \epsilon_{e q}^{m}$. According to $J_{2}$ deformation theory, the two equivalent quantities are related to the corresponding stress or strain components as, 


$$
\begin{aligned}
\sigma_{e q}^{m} & =\sqrt{\frac{1}{2}\left[\left(\sigma_{11}^{m}-\sigma_{22}^{m}\right)^{2}+\left(\sigma_{22}^{m}-\sigma_{33}^{m}\right)^{2}+\left(\sigma_{33}^{m}-\sigma_{11}^{m}\right)^{2}\right]+3\left(\sigma_{12}^{m^{2}}+\sigma_{13}^{m^{2}}+\sigma_{23}^{m^{2}}\right)} \\
\epsilon_{e q}^{m} & =\frac{1}{1+\nu_{s}^{m}} \sqrt{\frac{1}{2}\left[\left(\epsilon_{11}^{m}-\epsilon_{22}^{m}\right)^{2}+\left(\epsilon_{22}^{m}-\epsilon_{33}^{m}\right)^{2}+\left(\epsilon_{33}^{m}-\epsilon_{11}^{m}\right)^{2}\right]+\frac{3}{4}\left(\gamma_{12}^{m^{2}}+\gamma_{13}^{m^{2}}+\gamma_{23}^{m^{2}}\right)}
\end{aligned}
$$

where $\nu_{s}^{m}$ is the matrix secant Poisson's ratio defined by

$$
\nu_{s}^{m}=\frac{1}{2}+\frac{E_{s}^{m}}{E_{e}^{m}}\left(\nu_{e}^{m}-\frac{1}{2}\right)
$$

and $E_{e}^{m}$ and $\nu_{e}^{m}$ are the matrix elastic Young's modulus and elastic Poisson's ratio, respectively.

It can be shown that when the matrix is subjected to a uniaxial tension $\left(\sigma_{11}^{m}>0\right.$ and other $\left.\sigma_{i j}^{m}=0\right)$, $\sigma_{e q}^{m}$ is reduced to $\sigma_{11}^{m}$ according to Eq. (1). On the other hand, the non-zero strains in a uniaxial tensile stress state are $\epsilon_{11}^{m}>0$ and $\epsilon_{22}^{m}=\epsilon_{33}^{m}=-\nu_{s}^{m} \epsilon_{11}^{m}$. Substituting these relations into Eq. (2), $\epsilon_{e q}^{m}$ is readily reduced to $\epsilon_{11}^{m}$. Thus, a simple uniaxial stress-strain response from tensile testing can be used to construct the effective stress-strain response, and no other measurements are required providing a convenient way to capture pre-peak nonlinearity.

In this paper, the matrix nonlinear stress-strain relation is characterized using an exponential curve as,

$$
\sigma_{e q}^{m}=\sigma_{y}^{m}-\frac{K_{1}}{K_{2}}\left(e^{-K_{2} \epsilon_{e q}^{m}}-e^{-K_{2} \frac{\sigma_{y}^{m}}{E_{e}^{m}}}\right)
$$

where $\sigma_{y}^{m}$ is the yield stress in a simple uniaxial tension, and $K_{1}$ and $K_{2}$ are the two material parameters that govern the evolution of matrix microdamage which can be determined from an experimental stress-strain response. $^{24}$

\section{B. Modeling the Failure of Polymer Matrix Using Smeared Crack Approach}

The accumulation of matrix microdamage finally initiates matrix macroscopic cracking, followed by postpeak strain softening response in the stress versus strain curve. In this study, the evolution of matrix failure is modeled using the SCA, which is originally developed by Rots et al. ${ }^{25}$ to model crack propagation and fracture in concrete. In the SCA, it assumes that distributed cracks are "smeared" out over a certain width within the finite element such that the effect of progressive cracking is represented by a macroscopic strain softening behavior in a continuum scheme. To restore mesh objectivity, a characteristic length is introduced such that the total amount of energy dissipated during failure in a continuum element is equal to the fracture toughness defined for a cohesive element of the same size. The fracture toughness, or the critical energy release rate, $G_{C}$, is defined by the area under the traction-separation law that dictates the cohesive behavior of crack propagation (see Figure 11(b)) as,

$$
G_{C}=\int_{0}^{\delta_{f}} \sigma \mathrm{d} u
$$

where $u$ is the sum of crack displacements within the fracture zone, as schematically shown in Figure 11(a). In the SCA, $u$ represents the crack strain acting across a certain width within a finite element, denoted as the crack band width, $h$, which is shown in Figure 11(b). Assuming that all the cracks are uniformly distributed over the crack band, since $u$ is the accumulation of all the crack strains over the fracture zone, it gives,

$$
u=h \epsilon^{c r}
$$

If $g_{c}$ is defined as the area under the softening branch of the stress-strain curve, $g_{c}$ and $G_{C}$ are related by,

$$
G_{C}=h g_{c}
$$

Therefore, the strain-based description for a softening material is related to the displacement-based traction-separation laws through the characteristic length, $h$. In a FEA setting, $h$ is chosen based upon the element type, element size, element shape, and the integration scheme. ${ }^{25}$ Typically the length of the element projected onto the crack normal is used as a characteristic element length, as shown in Figure 13. 


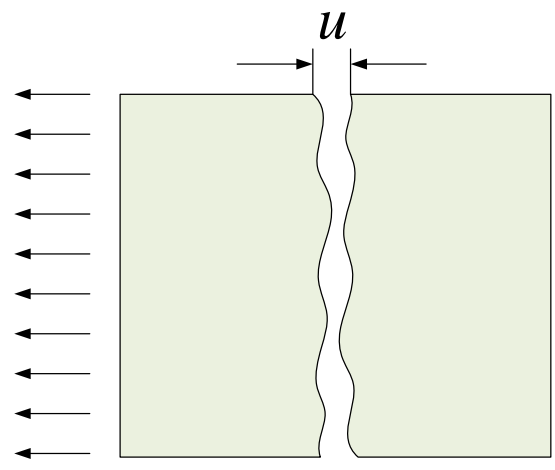

(a) Discrete crack.

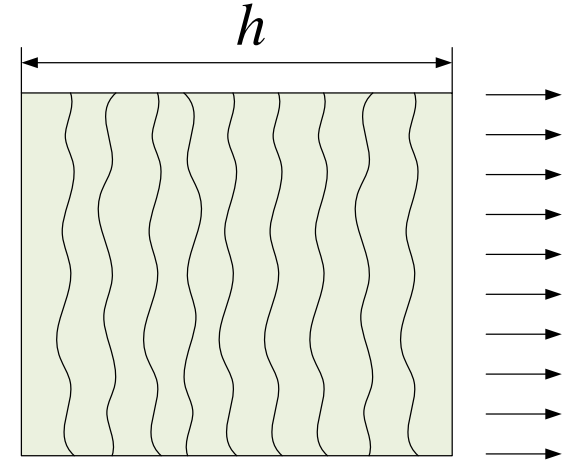

(b) Smeared crack.

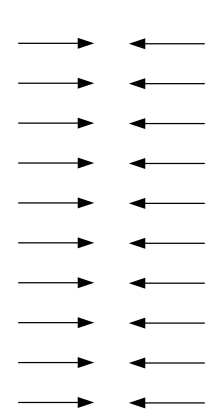

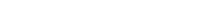

Figure 11. Discrete cracks are smeared out within a finite element.

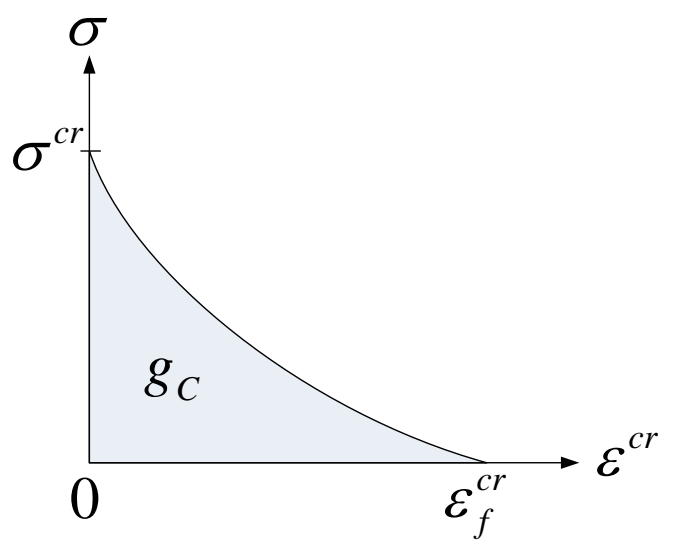

(a) Critical stress-strain curve.

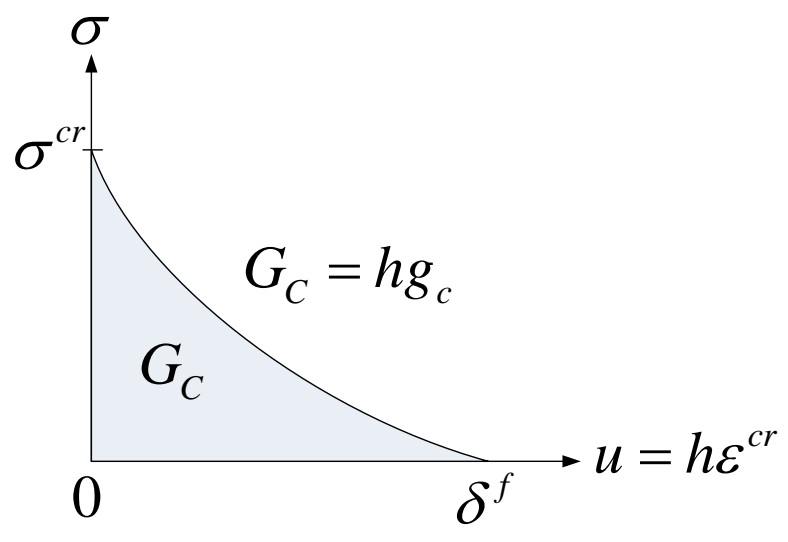

(b) Traction-separation law.

Figure 12. Stress-strain softening curve is related to traction-separation law through a characteristic length, $h$.

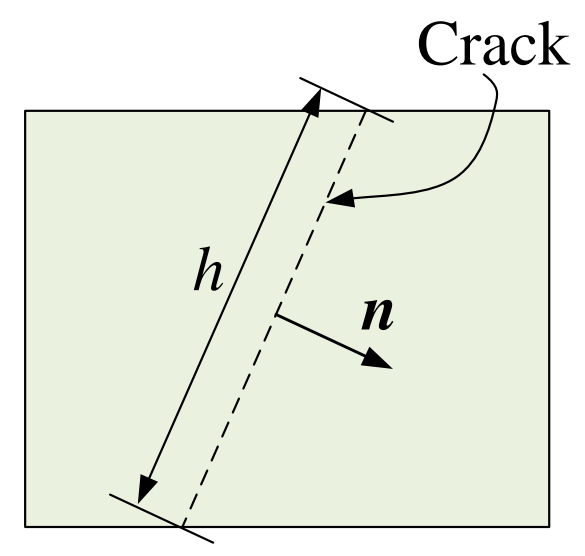

Figure 13. Characteristic element length. The length of the element projected on the crack normal is used as characteristic element length. 


\section{Formulations}

The formulation of the SCA for an isotropic material presented in this section is similar to that in. ${ }^{25,26}$ In the pre-peak regime, the material is governed by a standard continuum theory such as elasticity, plasticity, or CDM. When failure initiates, it is assumed that the total strains, $\boldsymbol{\epsilon}$, is decomposed into continuum strains, $\boldsymbol{\epsilon}^{c o}$, and cracked strains, $\boldsymbol{\epsilon}^{c r}$, as,

$$
\epsilon=\epsilon^{c o}+\epsilon^{c r}
$$

In a continuum scheme, $\epsilon^{c o}$ can be further decomposed into elastic, plastic, and thermal strains, if needed. In the current study, the matrix nonlinear response is modeled as a degrading secant solid, hence, the secant strains at the onset of failure initiation is used for $\boldsymbol{\epsilon}^{c o}$. Here, $\boldsymbol{\epsilon}, \boldsymbol{\epsilon}^{c o}$, and $\boldsymbol{\epsilon}^{c r}$ are presented in global coordinates.

Figure 14 shows the crack morphology in 3D. At the crack interface, there exist three relative displacements between the crack faces, one is the crack opening displacement, and the other two are the crack sliding displacements. The subscript $n$ and $t$ are used to designate directions normal to the crack and tangential to the crack, respectively. The key of the SCA is to embed cracks into a continuum, hence, the crack opening displacement is represented by a local normal crack strain, $\epsilon_{n n}^{c r}$, and the two crack sliding displacements are replaced by two local shear crack strains, $\gamma_{t 1}^{c r}$ and $\gamma_{t 2}^{c r}$. These local crack strains are defined in the local coordinates where the crack is oriented, and can be related to the global coordinates through a transformation matrix, $\boldsymbol{T}$, as,

$$
\boldsymbol{\epsilon}^{c r}=\boldsymbol{T} \boldsymbol{e}^{c r}=\boldsymbol{T}\left\{\begin{array}{l}
\epsilon_{n n}^{c r} \\
\gamma_{t 1}^{c r} \\
\gamma_{t 2}^{c r}
\end{array}\right\}
$$

where $\boldsymbol{e}^{c r}$ is a vector that contains local crack strains, and $\boldsymbol{T}$ is a 6 by 3 transformation matrix depending on the crack orientation. Similarly, the interface stresses at the crack interface, $\boldsymbol{s}^{c r}$, can be transferred to the global stress state, $\sigma$, through,

$$
\boldsymbol{s}^{c r}=\left\{\begin{array}{c}
\sigma_{n n}^{c r} \\
\tau_{t 1}^{c r} \\
\tau_{t 2}^{c r}
\end{array}\right\}=\boldsymbol{T}^{T} \boldsymbol{\sigma}
$$

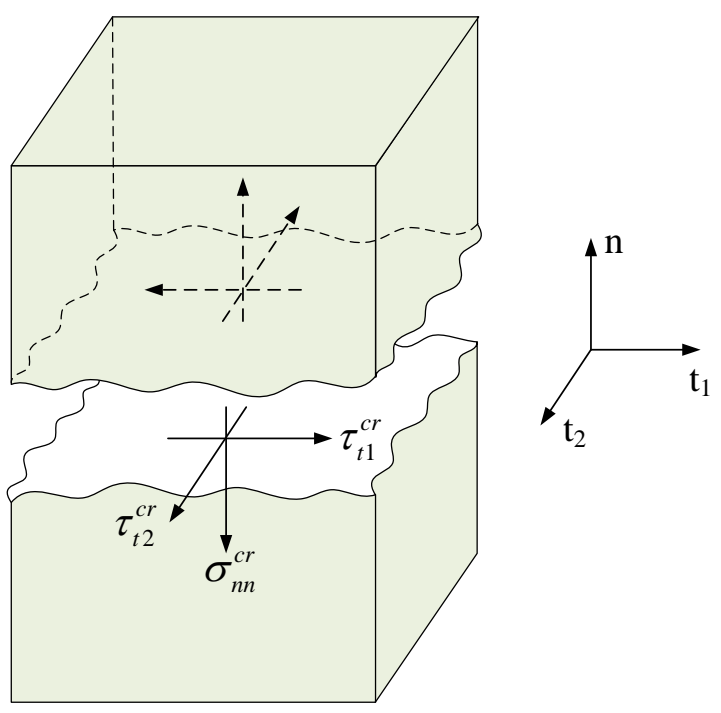

Figure 14. Crack morphology. There exist one normal and two shear crack components at the crack interface in $3 \mathrm{D}$ stress state. 
The crack interface stresses are related to the local crack strains through,

$$
s^{c r}=D^{c r} e^{c r}
$$

where $\boldsymbol{D}^{c r}$ is the crack interface stiffness matrix that dictates the failure evolution in the post-peak strain softening regime. For a single crack in a $3 \mathrm{D}$ solid, $\boldsymbol{D}^{c r}$ can be expanded as,

$$
\boldsymbol{D}^{c r}=\left[\begin{array}{ccc}
D_{c} & 0 & 0 \\
0 & G_{c 1} & 0 \\
0 & 0 & G_{c 2}
\end{array}\right]
$$

where $D_{c}$ is the secant stiffness across the crack interface due to crack opening, and $G_{c 1}$ and $G_{c 2}$ are the two secant shear stiffness governed by crack sliding. These quantities identify the modes of failure and are related to traction-separation laws with a characteristic length scale. The off-diagonal terms are assumed to be zero, indicating that there is no coupling between the normal and shear crack components. The coupling between the crack shear and opening displacements, known as crack dilatancy, has been extensively studied by Bažant and Gambarova, ${ }^{27}$ Walraven, ${ }^{28}$ Walraven and Reinhardt,${ }^{29}$ and Gambarova and Karakoç. ${ }^{30}$

It should be pointed out that the sudden loss of the positive definiteness of material global stiffness tensor may result in oscillations of the solutions in the post-peak softening regime. Hence, a damping matrix, $\boldsymbol{D}^{d a}$, is introduced to relate the stress-strain relation at the crack interface, and Eq. (11) becomes ${ }^{26}$

$$
\boldsymbol{s}^{c r}=\boldsymbol{D}^{c r} \boldsymbol{e}^{c r}+\boldsymbol{D}^{d a} \dot{\boldsymbol{e}}^{c r}
$$

Consequently, the crack progression becomes a time dependent property by the use of damping matrix. The crack strain rate, $\dot{e}^{c r}$, is approximated at each time step using backward finite difference scheme as,

$$
\dot{e}^{c r} \approx \frac{e^{c r}(t+\Delta t)-e^{c r}(t)}{\Delta t}=\frac{e^{c r}-e_{o l d}^{c r}}{\Delta t}
$$

The constitutive relation for a continuum is,

$$
\boldsymbol{\sigma}=\boldsymbol{D}^{c o} \boldsymbol{\epsilon}^{c o}
$$

where $\boldsymbol{D}^{c o}$ is the continuum stiffness tensor. In the current study, since the matrix material is dictated as a secant degrading solid in the pre-peak regime, $\boldsymbol{D}^{c o}$ is computed using the secant Young's modulus, $E_{s}$ and secant Poisson's ratio (see Eq. (3)). Combining Eq. (8), (9), (10), (13), (14), and (15), it gives the relation between the local crack strains and the total global strains can be obtained as,

$$
\boldsymbol{e}^{c r}=\left[\boldsymbol{D}^{c r}+\boldsymbol{T}^{T} \boldsymbol{D}^{c o} \boldsymbol{T}+\frac{1}{\Delta t} \boldsymbol{D}^{d a}\right]^{-1}\left[\boldsymbol{T}^{T} \boldsymbol{D}^{c o} \boldsymbol{\epsilon}+\frac{1}{\Delta t} \boldsymbol{D}^{d a} \boldsymbol{e}_{o l d}^{c r}\right]
$$

Finally, the relation between the total stress and total strain in the post-peak regime is computed as,

$$
\begin{aligned}
\boldsymbol{\sigma}= & {\left[\boldsymbol{D}^{c o}-\boldsymbol{D}^{c o} \boldsymbol{T}\left(\boldsymbol{D}^{c r}+\boldsymbol{T}^{T} \boldsymbol{D}^{c o} \boldsymbol{T}+\frac{1}{\Delta t} \boldsymbol{D}^{d a}\right)^{-1} \boldsymbol{T}^{T} \boldsymbol{D}^{c o}\right] \boldsymbol{\epsilon} } \\
& -\frac{1}{\Delta t} \boldsymbol{D}^{c o} \boldsymbol{T}\left[\boldsymbol{D}^{c r}+\boldsymbol{T}^{T} \boldsymbol{D}^{c o} \boldsymbol{T}+\frac{1}{\Delta t} \boldsymbol{D}^{d a}\right]^{-1} \boldsymbol{D}^{d a} \boldsymbol{e}_{\text {old }}^{c r}
\end{aligned}
$$

The details of the numerical implementation of the SCA within a FEA setting including the iteration scheme to determine the crack strains are discussed by Heinrich and Waas. ${ }^{26}$

\section{Determination of the Crack Constitutive Relations}

In the present study, 1D traction-separation laws are employed, consequently, Eq. (12) becomes,

$$
\boldsymbol{D}^{c r}=\left[\begin{array}{ccc}
E^{c r} & 0 & 0 \\
0 & G_{1}^{c r} & 0 \\
0 & 0 & G_{2}^{c r}
\end{array}\right]
$$


where $E^{c r}$ is the secant crack modulus resulting from normal crack strain (mode-I type of failure), while $G_{1}^{c r}$ and $G_{2}^{c r}$ are associated with shear crack strains (mode-II type of failure). In the current study, secant crack stiffness is used so that the softening response follows the traction-separation laws exactly, as shown in Figure 15. To prevent crack healing, it is required that,

$$
\dot{E}^{c r}<0 \text { and } \dot{G}^{c r}<0
$$

Hence, once the crack stiffness is degraded, it cannot be recovered. The loading and unloading behavior during the evolution of the failure process is specified in Figure 15.

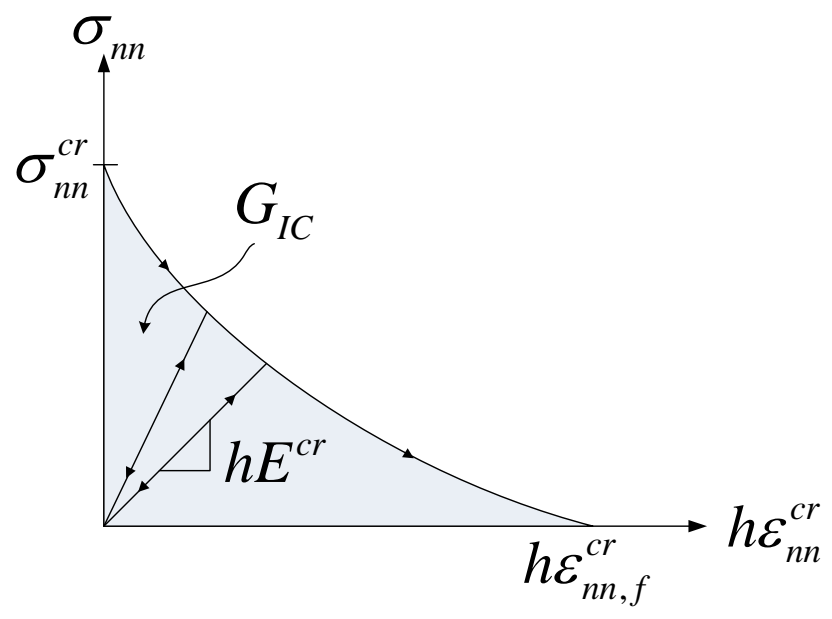

Figure 15. Crack evolution is dictated using degrading secant crack stiffness.

Matrix tensile cracking has been observed in the experiment. In a monolithic material, cracks are likely to grow under pure mode-I conditions since this type of failure mode is energetically favorable. In 3DTCs, the cracks in the surrounding matrix may be subjected to mixed-mode loading due to the presence of fiber tows, or at microscale, the crack orientation of the matrix inside the fiber tow can be affected by the individual fibers. However, in this study, it is assumed that the tensile cracks grow under pure mode-I condition, aligned with the maximum principal stress plane. For a given stress state, the principal stresses, $\sigma_{1}, \sigma_{2}$, and $\sigma_{3}$, and the corresponding principal axes $\boldsymbol{n}_{\mathbf{1}}, \boldsymbol{n}_{\mathbf{2}}$, and $\boldsymbol{n}_{\mathbf{3}}$, are first computed. Maximum tensile stress criterion is used for tensile crack initiation as,

$$
\sigma_{1}>\sigma_{2}>\sigma_{3} \text { and } \frac{\sigma_{1}}{\sigma^{c r}}=1
$$

where $\sigma^{c r}$ is the cohesive strength, or the critical stress, in the mode-I traction separation law. In practical applications, it is further assumed that once a crack is initiated, the crack orientation, determined from the principal stress directions, is fixed during the failure evolution. It is further assumed that the crack interface is traction free in both normal and shear directions if all the fracture energy has been dissipated. The two crack shear moduli, $G_{1}^{c r}$ and $G_{2}^{c r}$, are degraded as a function of $\epsilon_{n n}^{c r}$, indicating that the cracks grow under mode-I dominated conditions. It is possible that the crack shear moduli are degraded with respect to $\gamma_{t 1}^{c r}$ or $\gamma_{t 2}^{c r}$, and a mixed-mode traction-separation law should be introduced to ensure that the shear failure evolves under mode-II conditions. ${ }^{31}$ However, such a complicated failure mechanism requires further study of the crack progression at the microscale, and as such this is not considered in the current study. Matrix nonlinear and fracture properties are provided in Table 3.

Table 3. Damage and failure characteristics of SC-15 matrix.

\begin{tabular}{ccccc}
\hline$\sigma_{y}^{m}(\mathrm{MPa})$ & $K_{1}(\mathrm{MPa})$ & $K_{2}$ & $\sigma^{c r}(\mathrm{MPa})$ & $G_{I C}(\mathrm{~N} / \mathrm{mm})$ \\
\hline 25 & 1700 & 40 & 50 & 1.5 \\
\hline
\end{tabular}




\section{Fiber Tow Constitutive Model}

In 3DTCs, a singer fiber tow consists of thousands of individual fibers embedded in the surrounding matrix medium, the microstructure of which can be represented as a unidirectionally aligned fiber-reinforced composite. When the fiber tow undulates along its longitudinal direction, each infinitesimal section of a fiber tow can be considered as a unidirectional composite, with its local coordinates aligned with the tow orientation, ${ }^{4}$ as schematically shown in Figure 16. Hence, computing the fiber tow stress versus strain relation is equivalent to establishing a constitutive model for a unidirectional composite. The evolution of matrix microdamage (discussed in Section V.A) at the microscale manifest as the degradation of the fiber tow stiffness at the mesoscale, resulting in a pre-peak nonlinear stress-strain relation that can be captured using a two-scale model established by Zhang and Waas. ${ }^{32}$ In their model, the subscale micromechanical analysis on the fiber and matrix is carried out in close form, and the evolution of the fiber tow nonlinear response is assumed to be governed by two strain based variables that are related the extreme values of an appropriately defined matrix equivalent strain. The implementation of the two-scale model is presented in Section VI.A.

In the experiment, catastrophic failure mechanisms have been observed, including fiber tow breakage, tow kinking, and transverse cracking, resulting in a loss of load carrying capability at the macroscale, followed by a post-peak strain softening response in the stress versus stress curve. Since the positive definiteness of the tangent stiffness matrix is lost, the aforementioned two-scale model will provide mesh dependent results in a FEA framework if no characteristic length is introduced. Therefore, the SCA established in Section V.B is suitable for modeling the tow failure behavior, as presented in Section VI.B.

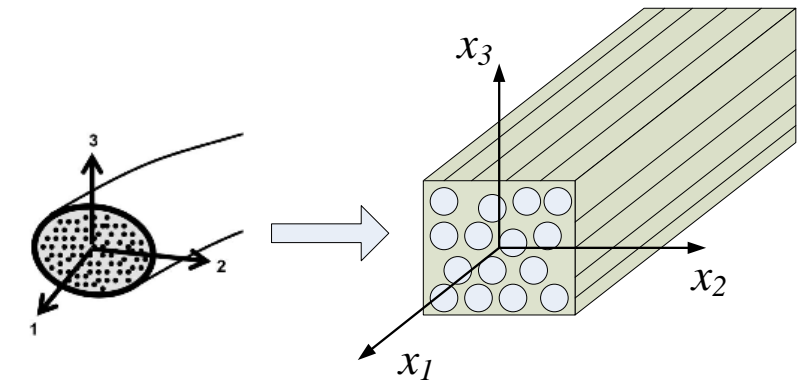

Figure 16. Fiber tow is represented as a unidirectional fiber-reinforced composite.

\section{A. Pre-peak Nonlinear Response}

\section{Microstructure of Fiber Tow}

The microstructure of a single fiber tow is shown in Figure 16, in which the fibers are assumed to be infinitely long with a statistically isotropic plane that is transverse to the fiber direction. The effective response of such a material is transversely isotropic, requiring five independent constants to form the composite stiffness tensor. Although the choices of these elastic constants are not unique, the axial modulus, $E_{1}^{t}$, the axial Poisson's ratio, $\nu_{12}^{t}$, the axial shear modulus, $G_{12}^{t}$, the plane-strain bulk modulus, $K_{23}^{t}$, and the transverse shear modulus, $G_{23}^{t}$, are used throughout the paper. Therefore, the stiffness tensor for a transversely isotropic fiber tow can be written in terms of these elastic constants as,

$$
\boldsymbol{C}^{t}=\left[\begin{array}{cccccc}
E_{1}^{t}+4 \nu_{12}^{t^{2}} K_{23}^{t} & 2 \nu_{12}^{t} K_{23}^{t} & 2 \nu_{12}^{t} K_{23}^{t} & 0 & 0 & 0 \\
2 \nu_{12}^{t} K_{23}^{t} & K_{23}^{t}+G_{23}^{t} & K_{23}^{t}-G_{23}^{t} & 0 & 0 & 0 \\
2 \nu_{12}^{t} K_{23}^{t} & K_{23}^{t}-G_{23}^{t} & K_{23}^{t}+G_{23}^{t} & 0 & 0 & 0 \\
0 & 0 & 0 & G_{23}^{t} & 0 & 0 \\
0 & 0 & 0 & 0 & G_{12}^{t} & 0 \\
0 & 0 & 0 & 0 & 0 & G_{12}^{t}
\end{array}\right]
$$

Other important constants, including the transverse modulus, $E_{2}^{t}$, and transverse Poisson's ratio, $\nu_{23}^{t}$, can 
be computed as,

$$
\begin{aligned}
E_{2}^{t} & =\frac{4 G_{23}^{t} K_{23}^{t}}{K_{23}^{t}+\psi G_{23}^{t}} \\
\nu_{23}^{t} & =\frac{K_{23}^{t}-\psi G_{23}^{t}}{K_{23}^{t}+\psi G_{23}^{t}}
\end{aligned}
$$

where,

$$
\psi=1+\frac{4 K_{23}^{t} \nu_{12}^{t^{2}}}{E_{1}^{t}}
$$

In the pre-peak nonlinear regime, the fiber is assumed to be linear elastic, transversely isotropic, with "1" designating its longitudinal direction. Its stiffness tensor, $\boldsymbol{C}^{f}$, can be written in terms of fiber properties using Eq. (21) by replacing the superscript "t" with "f". The matrix material is an isotropic elastic-damaging solid, and its nonlinear response is modeled using a modified $J_{2}$ deformation theory of plasticity through a secant moduli approach, as discussed in Section V.A. As a result, the composite effective stress-strain response is extended to the nonlinear regime by substituting secant moduli into Eq. (21).

\section{Micromechanics Model}

The key to the micromechanics model proposed by Zhang and Waas ${ }^{32}$ is to relate the fiber tow strains (the strain applied on the fiber-matrix microstructure) to the local matrix strain fields through a 6 by 6 transformation matrix, $\boldsymbol{F}$, as,

$$
\left\{\begin{array}{l}
\epsilon_{11}^{m} \\
\epsilon_{22}^{m} \\
\epsilon_{33}^{m} \\
\gamma_{12}^{m} \\
\gamma_{13}^{m} \\
\gamma_{23}^{m}
\end{array}\right\}=\left[\begin{array}{llllll}
F_{11} & F_{12} & F_{13} & F_{14} & F_{15} & F_{16} \\
F_{21} & F_{22} & F_{23} & F_{24} & F_{25} & F_{26} \\
F_{31} & F_{32} & F_{33} & F_{34} & F_{35} & F_{36} \\
F_{41} & F_{42} & F_{43} & F_{44} & F_{45} & F_{46} \\
F_{51} & F_{52} & F_{53} & F_{54} & F_{55} & F_{56} \\
F_{61} & F_{62} & F_{63} & F_{64} & F_{65} & F_{66}
\end{array}\right]\left\{\begin{array}{c}
\epsilon_{11}^{t} \\
\epsilon_{22}^{t} \\
\epsilon_{33}^{t} \\
\gamma_{12}^{t} \\
\gamma_{13}^{t} \\
\gamma_{23}^{t}
\end{array}\right\}
$$

The $F_{i j}$ components can be computed by imposing a single non-zero composite strain on the fiber-matrix microstructure and solving the resulting matrix strain fields. In particular, the axial properties, including the axial tension $\left(E_{1}^{t}\right.$ and $\left.\nu_{12}^{t}\right)$ and axial shear $\left(G_{12}^{t}\right)$, are computed through a two-phase concentric cylinder model $(\mathrm{CCM}),{ }^{33}$ which is subsequently used for the computation of $F_{i 1}, F_{i 4}$, and $F_{i 5}$. The rest of the components in the $\boldsymbol{F}$ matrix are determined via an extended three-phase generalized self-consistent model $(\mathrm{GSCM}),{ }^{34}$ which also gives the composite transverse properties, $K_{23}^{t}$ and $G_{23}^{t}$. The computation of each component in the $\boldsymbol{F}$ matrix as well the composite effective constants are provided in Appendix. It should be noted that the proposed micromechanics model is based upon the homogenization techniques that are originally used to compute the composite effective moduli, hence, both the fiber and matrix are assumed to be linear elastic. When the matrix stiffness is reduced due to microdamage, the nonlinear response of the matrix is modeled through a secant moduli approach, in which the matrix elastic properties are replaced with the corresponding secant moduli.

\section{Modeling Methodology for Nonlinear Fiber Tow}

The modeling methodology for nonlinear fiber tow is based upon two scales. The mesoscale tow-level analysis is conducted by utilizing effective homogenized properties to compute the local fields in each fiber tow. Simultaneously, it is intended to carry out the micromechanical analysis, at the fiber and matrix level, using the micromechanics model presented in Section VI.A.2, in which the constituent stress and strain fields are provided in close form. The commercially available finite element software, ABAQUS (version 6.12), is used for the mesoscale FEA, and the micromechanics model at the subscale is implemented at each integration point of the mesoscale model, using a user defined material subroutine, UMAT. This subroutine is called at each integration point at each increment, and the material constitutive law is updated through user-defined options.

At the start of each increment, the fiber tow states (stress, strain, and solution-dependent state variables) from the previous equilibrium step and the strain increments in the current step are passed into the 
UMAT through the ABAQUS solver. In the $n$th increment, the total strain, $\epsilon_{i j}^{n}$, is calculated by adding the current strain increment, $d \epsilon_{i j}^{n}$, to the strain in the previous step, $\epsilon_{i j}^{n-1}$, as $\epsilon_{i j}^{n}=\epsilon_{i j}^{n-1}+d \epsilon_{i j}^{n}$. In the multiscale computational scheme, the strains at each integration point in the FEA, are applied to the subscale micromechanics model. These integration point strains can be treated as the effective tow strains that are applied on a discrete fiber-matrix microstructure. The constituent strain fields therefore can be computed in close form by knowing the globally applied strains through the $\boldsymbol{F}$ matrix in Eq. (24).

It should be noted that the resulting matrix strain fields vary in space, ${ }^{32}$ hence the matrix equivalent strain, computed using Eq. (2), has spatial variation as well. In the current fully analytical computational scheme, it is hypothesized that the composite nonlinear behavior can be characterized using two scalar variables that are related to the matrix equivalent strain. This idea is similar to the mean-field theories in which the average value of the strain fields are utilized to determine the matrix nonlinear progression. In the present study, the two scalar variables that govern the evolution of matrix microdamage are defined based upon the maximum and average value of the square of the matrix equivalent strain at the fiber-matrix interface, respectively, as,

$$
\begin{aligned}
V_{\text {max }} & =\max \left\{\frac{1}{2}\left[\left(\bar{\epsilon}_{11}^{m}-\bar{\epsilon}_{22}^{m}\right)^{2}+\left(\bar{\epsilon}_{22}^{m}-\bar{\epsilon}_{33}^{m}\right)^{2}+\left(\bar{\epsilon}_{33}^{m}-\bar{\epsilon}_{11}^{m}\right)^{2}\right]+\frac{3}{4}\left(\bar{\gamma}_{12}^{m^{2}}+\bar{\gamma}_{13}^{m^{2}}+\bar{\gamma}_{23}^{m^{2}}\right)\right\} \\
V_{\text {avg }} & =\operatorname{avg}\left\{\frac{1}{2}\left[\left(\bar{\epsilon}_{11}^{m}-\bar{\epsilon}_{22}^{m}\right)^{2}+\left(\bar{\epsilon}_{22}^{m}-\bar{\epsilon}_{33}^{m}\right)^{2}+\left(\bar{\epsilon}_{33}^{m}-\bar{\epsilon}_{11}^{m}\right)^{2}\right]+\frac{3}{4}\left(\bar{\gamma}_{12}^{m^{2}}+\bar{\gamma}_{13}^{m^{2}}+\bar{\gamma}_{23}^{m^{2}}\right)\right\}
\end{aligned}
$$

where $\bar{\epsilon}_{i j}^{m}$ is the matrix strain at the fiber-matrix interface. Physically, the average term is dominant in the matrix strain field when the fiber volume fraction is low, while the maximum value dominates the result for high fiber volume fraction. Therefore, a weight function of $V_{\max }$ and $V_{\text {avg }}$ can be written as,

$$
V_{\text {weight }}=V_{f}^{n} V_{\max }+\left(1-V_{f}^{n}\right) V_{\text {avg }}
$$

where $n$ is dependent on the fiber-to-matrix stiffness ratio such that the effect of constituent properties can be accounted for. In this paper, it is assumed that,

$$
n=2 \sqrt{\left(\frac{E^{m}}{E_{2}^{f}}+\frac{G^{m}}{G_{23}^{f}}\right)}
$$

Consequently, two matrix equivalent strains can be computed; one is based upon the weight function in Eq. 27 , while the other is based upon the average value in Eq. (26), as,

$$
\epsilon_{m, 1}^{e q}=\frac{1}{1+\nu_{s}} \sqrt{V_{w e i g h t}} \quad \text { and } \quad \epsilon_{m, 2}^{e q}=\frac{1}{1+\nu_{s}} \sqrt{V_{a v g}}
$$

In the present study, since the fiber is significantly stiffer than the matrix material, $V_{\text {weight }} \approx V_{\text {max }}$. Once the matrix equivalent strain is resolved, the matrix stiffness tensor is degraded as a secant solid according to the nonlinear damage model presented in Section V.A. It is further hypothesized that the matrix secant moduli calculated using $\epsilon_{m, 1}^{e q}$ are subsequently used to compute the tow effective secant moduli, $E_{1}^{t}, \nu_{12}^{t}, K_{23}^{t}$, and $G_{23}^{t}$ using Eq. (30), (31), (33), and (34), respectively; whereas the matrix secant moduli determined from $\epsilon_{m, 2}^{e q}$ is used to compute $G_{12}^{t}$ using Eq. (32). Consequently, if matrix microdamage occurs, the stiffness of the subscale microstructure is reduced based upon the proposed secant moduli approach. The subscale stiffness tensors are subsequently used to update the fiber tow stiffness and stresses in the mesoscale FEA. Therefore, the influence of matrix microdamage at the subscale manifests as the degradation of the tow stiffness in the mesoscale model discussed in Section IV.

\section{B. Post-peak Failure Response}

In 3DTCs, fiber tows are surrounded by polymer matrix material, resulting in a complicated state of stress. Due to the heterogeneity of the microstructure and the complexity of the local fields, fiber tows exhibit multiple failure modes, including tow kinking in compression, tow breakage in tension, shear banding, and transverse and shear cracking. In some instance, the fiber tow can be delaminated from the surrounding matrix at high strain rates, as shown by Pankow et al. ${ }^{35}$ through a split Hopkinson pressure bar test. Shear bands are observed when the textile composite is subjected to the through-the-thickness compression. ${ }^{20,35}$ 
Cracks that grow along the transverse normal, axial shear, and transverse shear directions are considered as the matrix failure mode, dominated by the strength and toughness of the polymer matrix material.

In the present study, fiber tow kinking and breakage are considered as the two major failure modes for 3D textile composites subjected to flexural loading, which is evident from the experimental investigation discussed in Section III. Since it is difficult to experimentally measure the compressive strength of a fiber tow, a micromechanical analysis based upon the two-scale model proposed in Section VI.A is carried out to determine the compressive strength of a fiber tow, as presented in Section VI.B.1. The obtained compressive strength is used as the failure initiation criterion for a fiber tow subjected to compression. The progressive failure is modeled within a FEA framework as demonstrated in Section VI.B.2.

\section{Compressive Failure: A Micromechanical Study on Kink Banding}

The compressive strength of a unidirectional fiber-reinforced composite is significantly lower than the tensile strength due to the unique failure mechanism of kink banding. Since the load carrying capability has been limited by the compressive strength, kinking has been determined as a strength controlling mechanism of failure for this type of materials. This critical failure mode has been extensive studied by a number of researchers, such as Budiansky and Fleck, ${ }^{13}$ Kyriakides et al., ${ }^{14}$ Schultheisz and Waas, ${ }^{36}$ Waas and Schultheisz,${ }^{37}$ Lee and Waas, ${ }^{15}$ Basu and Waas, ${ }^{38}$ Feld et al. ${ }^{16,39}$ and Prabhakar and Waas. ${ }^{17,} 18$ The main physical event associated wit the kink band formation is the rotation of the fibers in a band within a degrading matrix. The rotation of fibers gives a rise to localized shear strains that drive the shear degradation of the matrix material between the fibers. The shear degradation in turn increases the rotation of the fibers, creating a positive feedback loot that culminates in a limit-load type instability. It is important to note that the formation of kink banding is an evolutionary event that is driven by the external loading with the evolutionary parameters being the band rotation and the fiber rotation within the bands.

Since kink banding occurs at the fiber level, it is motivated to model the evolution of kinking through a full micromechanics FEA model, as presented by Kyriakides et al.,${ }^{14}$ Vogler et al., ${ }^{40} \mathrm{Hsu}$ et al., ${ }^{41}$ and Yerramalli and Waas. ${ }^{42}$ These FEA models containing individual fibers and surrounding matrix provide deep understanding on the kink band formation and the subsequent evolution process. On the other hand, a more computationally efficient model on the lamina level (or tow-level) has been developed to predict the kinking strength. In these models, the influence of matrix nonlinearity at the microscale manifests as the nonlinear stress versus strain relation of the lamina at the mesoscale. The lamina nonlinear response can be obtained either from simple tension and shear tests ${ }^{23}$ or from a micromechanics based computational model. The nonlinear progression can be dictated through a nonlinear constitutive law, such as Schapery theory ${ }^{43}$ or Hill's anisotropic plasticity theory. ${ }^{44}$ The direct implementation of the lamina level nonlinear constitutive relations within a FEA framework to predict the kind band formation has been presented by Basu et al., ${ }^{38}$ Song et al., ${ }^{45}$ and Zhang et al. ${ }^{10}$

In this paper, the two-scale model established in Section VI.A is used to predict the kinking strength of a fiber tow. The details of the procedure are provided in Refs. ${ }^{38,45,10}$ The predicted kinking strength for the three different fiber misalignment angles are summarized in Table 4. The kinking strength of $592 \mathrm{MPa}$, which results from an initial fiber misalignment of $1.5^{\circ}$, is used as the fiber tow compressive strength in the subsequent failure analysis.

Table 4. Computed tow kinking strength using various initial fiber misalignment angles.

\begin{tabular}{cccc}
\hline Misalignment Angle & $1^{\circ}$ & $1.5^{\circ}$ & $2^{\circ}$ \\
\hline Kinking Strength (MPa) & 741 & 592 & 497 \\
\hline
\end{tabular}

\section{Implementation of the SCA to Model the Failure Response of a Single Fiber Tow}

Failure analysis of a single fiber tow requires micromechanical consideration to couple the influence of microstructure on the macroscale response. Therefore, performing failure analysis on the fiber and matrix level is motivated. The transverse compression and shear of a unidirectional fiber-reinforced composite have been studied by Totry et al. ${ }^{46,47,48}$ through a micromechanics FEA model. Recently, Pineda et al. ${ }^{49}$ studied the progressive failure behavior of a unidirectional lamina subjected to transverse tension and compression by implementing the crack band theory within the high-fidelity generalized method of cells model. These 
computational micromehanics models provide better understanding on the failure progression of a composite material, however, they are computationally expansive, and are not suitable to be implemented within a fiber tow of a 3D textile composite. Alternatively, phenomenological failure criteria can be utilized to predict the macroscopic failure response of a unidirectional composite or a single fiber tow by assuming the existence of certain types of failure modes. This work was pioneered by Hashin, ${ }^{50}$ and was later improved and implemented within a FEA framework by a number of researchers ${ }^{51,52,31,53}$ to demonstrate the predictive capability of this methodology. The use of phenomenological failure criteria offers distinct computational efficiency compared with the micromechanics model, while in the latter, the physics of the failure mechanism is preserved.

The SCA formulated in Section V.B is utilized to model the failure progression of a single fiber tow. Two major failure modes, compressive kinking and tensile breakage, are considered as the two major failure modes in this paper. It further assumes that when the critical stress (either tension or compression) is reached, the crack plane aligns perpendicular to the fiber direction. Therefore, the crack orientation transformation matrix, $\boldsymbol{T}$, is determined by the material orientation rather than the state of stress. Since a fiber tow can fail either in tension or compression, a specific traction-separation law is designed as illustrated in Figure 17. It is worth noting that when the failure progresses in compression, the stress at the crack interface does not drop completely. In the experiment, the formation of kink band limits the peak load, while additional kink bands are developed with continued deformation, resulting in a "load plateau". It indicates that even though fibers are broken within the kink band, load are still transferred through the band, allowing for stress redistribution. In this study, it is assumed that the crack interface can carry $50 \%$ of the kinking strength. Study on the post-kinking response is motivated to determine the amount of the load that can be transferred after kink band formation. ${ }^{54}$ The failure characteristics used in the FEA model are given in Table 5. The tensile strength is taken from Ref. ${ }^{55}$

Table 5. Failure characteristics of fiber tow.

\begin{tabular}{ccc}
\hline & $\sigma^{c r}(\mathrm{MPa})$ & $G_{I C}(\mathrm{~N} / \mathrm{mm})$ \\
\hline Tension & 1900 & 200 \\
\hline Compression & 572 & 10 \\
\hline
\end{tabular}

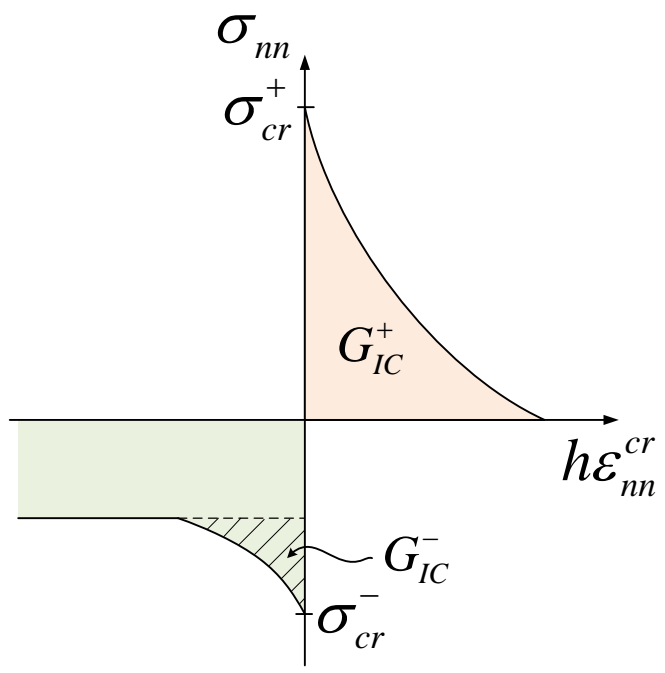

Figure 17. Traction-separation law for fiber tow. 


\section{Results and Discussion}

The orthotropic material properties for the homogenous model in the far field are calculated using the analytical model developed by Quek et al., ${ }^{4}$ and the material constants are summarized in Table 6 . The subscripts "x", "y", and "z" designate the axes illustrated in Figure 1. The elastic and fracture properties for the fiber and matrix are provided in Table 1, 3, and 5. The length of the mesoscale model is $40 \mathrm{~mm}$ so that the damage and failure modes can be fully developed within in the RUCs. The analysis is carried out using dynamic implicit method in ABAQUS standard solver.

Table 6. Homogeneous properties of the macroscale model. $x, y$, and $z$ designate the axes shown in Figure 1.

\begin{tabular}{ccccccccc}
\hline$E_{x}$ & $E_{y}$ & $E_{z}$ & $G_{x z}$ & $G_{x y}$ & $G_{z y}$ & $\nu_{x z}$ & $\nu_{x y}$ & $\nu_{z y}$ \\
$\mathrm{GPa}$ & $\mathrm{GPa}$ & $\mathrm{GPa}$ & $\mathrm{GPa}$ & $\mathrm{GPa}$ & $\mathrm{GPa}$ & - & - & - \\
\hline 19.0 & 5.34 & 16.7 & 2.32 & 2.63 & 3.20 & 0.06 & 0.35 & 0.46 \\
\hline
\end{tabular}

The computed load-displacement curve for the specimen loaded along the warp direction is compared against the experimental results, as shown in Figure 18. The proposed model offers good prediction on the elastic flexural stiffness, kink band initiation and progression. However, the computed result shows an increase in load for the deformation between $9.4 \mathrm{~mm}$ and $12.3 \mathrm{~mm}$ (between point (4) and (5) in Figure 18), while in the experiment the load is still in the plateau regime. The fiber tow progressive failure response is shown in Figure 20, where the color contours illustrate the absolute values of tow crack strains. Since the fiber tow is modeled using the SCA, the crack strains indicate kink band formation on the compression side and tow breakage on the tension side. As shown in Figure 20, the predicted "load plateau" between point (2) and (4) dictates the progression of kink banding on the compression side. Even though kink bands continue to progress between point (4) and (5), the resulting load increases with increased deformation. One possible reason is the ignorance of nonlinearity in the macroscale model. Although no visible damage and failure modes were reported in these areas according to the experiment, the homogenized parts can experience nonlinear response due to large deformation. Another possible reason for this artificial load increase is that the shear failure in fiber tow is ignored when the fiber tow is subject to tension. The coupling effect between the shear and tension failure of a fiber tow is currently under investigation. A significant load drop occurs at point (6), where the bottom fiber tow breakage is observed, as shown in Figure 20(f). The ABAQUS standard solver encounters convergence difficulty after point (6). The evolution of matrix cracking is shown in Figure 19.

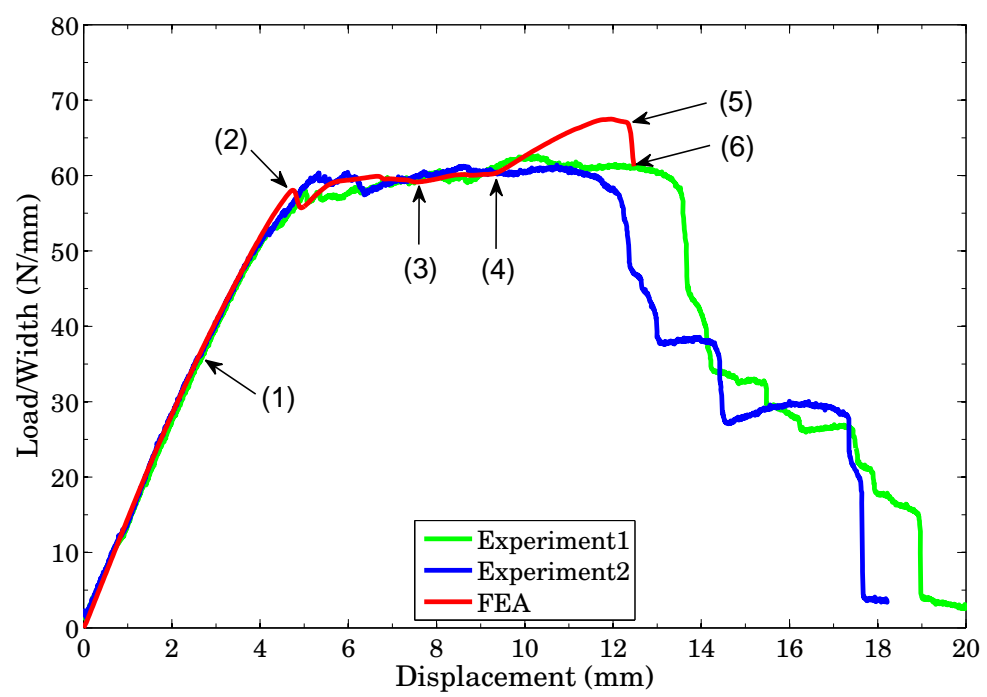

Figure 18. Comparison of the load-displacement curves obtained from the experiment and the computational model. The specimen is loaded along the warp direction. 
The difficulty in the analysis of 3DTCs lies in the fact that it is hard to individually characterize the constitutive response of each constituent (fiber tows and surrounding polymer matrix) within in the composite, while the macroscale structure fails progressively, coupled with multiple damage and failure modes. Therefore, the proposed model is useful in understanding the influence of the constituent properties on the macroscale response of the 3DTCs. Work is continuing to investigate the effect of failure characteristics, including the tensile and compressive strength of the fiber tow, and the associated fracture toughness, on the predicted flexural response of the 3DTCs.

\section{Conclusions}

The flexural response of a 3D layer-to-layer orthogonal interlocked textile composite has been examined experimentally and modeled using a multiscale FEA based mechanics model. Fiber tow kinking on the compressive side of the flexed beams has been found to be a strength limiting mechanism for both warp and weft specimens. Surface strain patterns, using digital image correlation show that tensile matrix cracking is initiated prior to the peak load in the warp direction loaded specimens.

A multiscale computational model, in which the detailed textile architecture is modeled at the mesoscale, has been developed to capture the observed damage and failure characteristics. The pre-peak nonlinear response of the fiber tow is modeled using a novel, two-scale model, in which the subscale micromechanical analysis on the fiber and matrix is carried out in close form. The influence of the matrix microdamage at the microscale manifests as the progressive degradation of the fiber tow stiffness at the mesoscale. The post-peak strain softening responses of the fiber tows and the surrounding polymer matrix are modeled through the SCA. The load-deflection response, along with the observed damage events, including matrix cracking, tow kinking, and tow tensile breakage, are successfully captured through the proposed model. Since all the inputs are from the constituent level, the model is useful in understanding how the 3DTC response is influenced by the geometry and textile architecture, the constitutive response of the constituents, and details of any unintended geometrical deviations of the textile architecture. 
(a) At point (1)

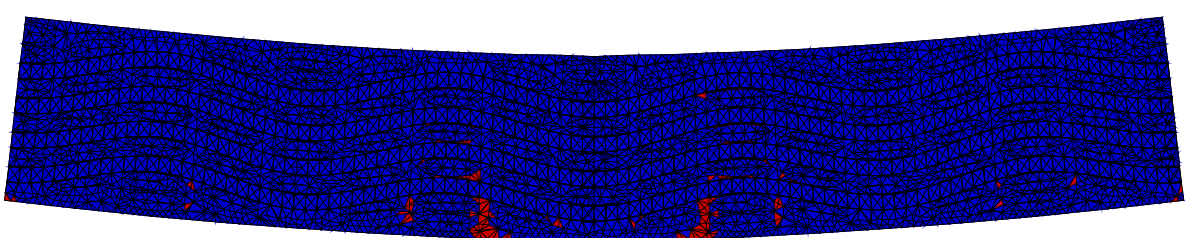

(b) At point (2)

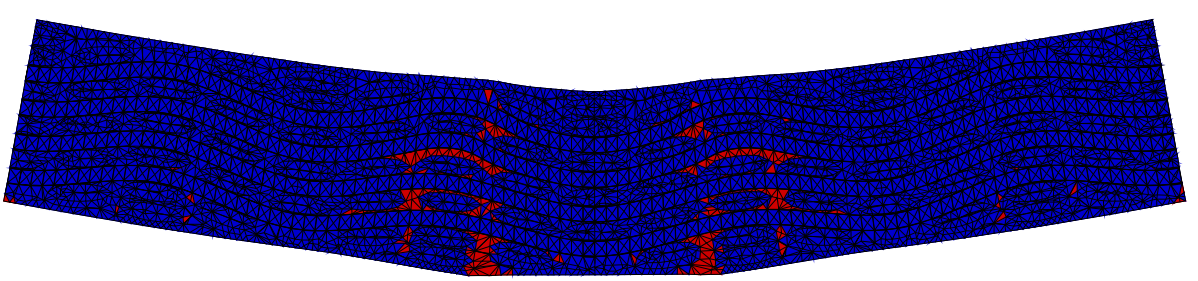

(c) At point (3)

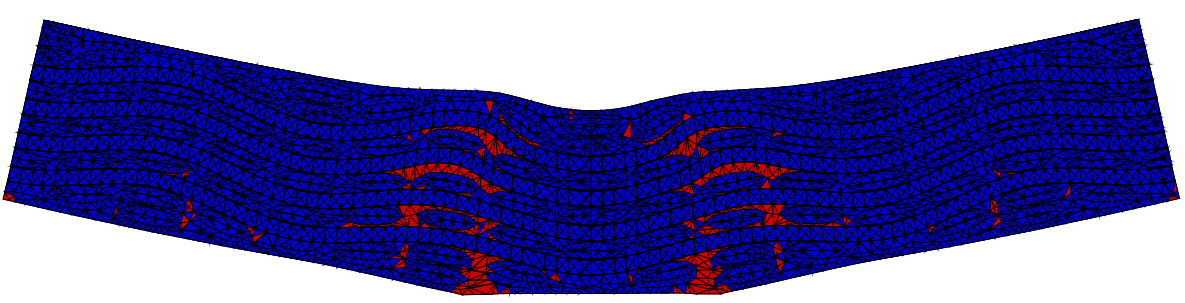

(d) At point (4)

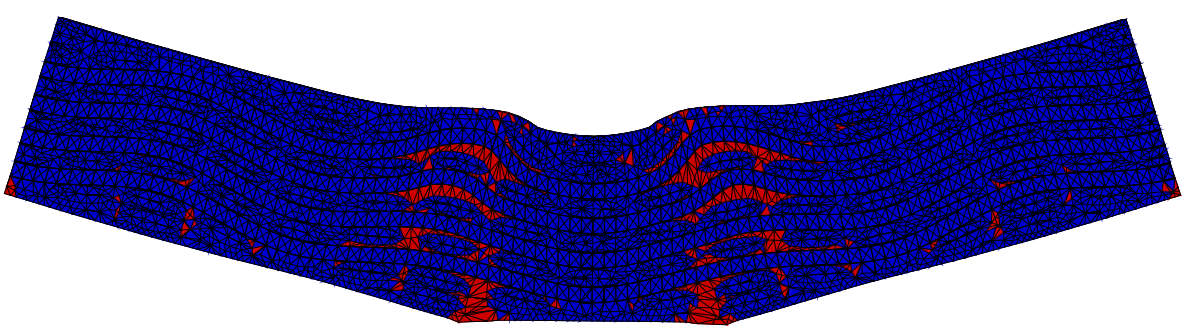

(e) At point (5)

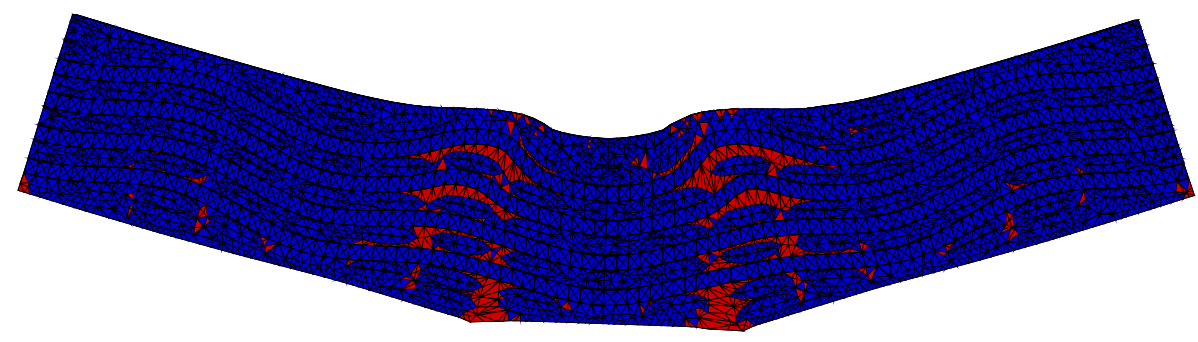

(f) At point (6)

Figure 19. Evolution of matrix cracking during the deformation. The cracked elements are shown in red. 

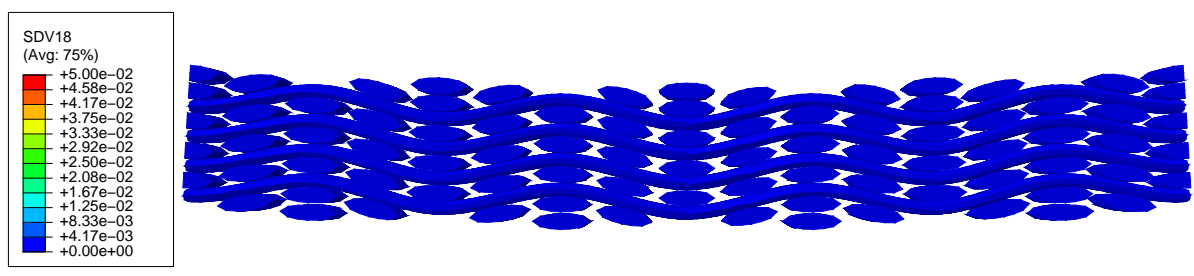

(a) At point (1)

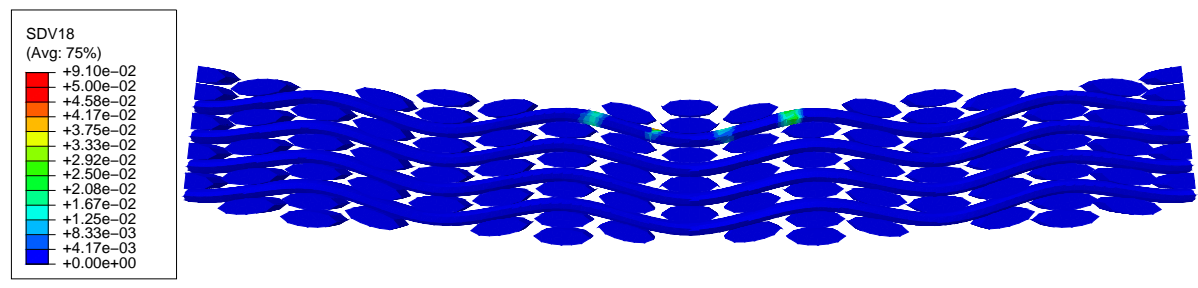

(b) At point (2)

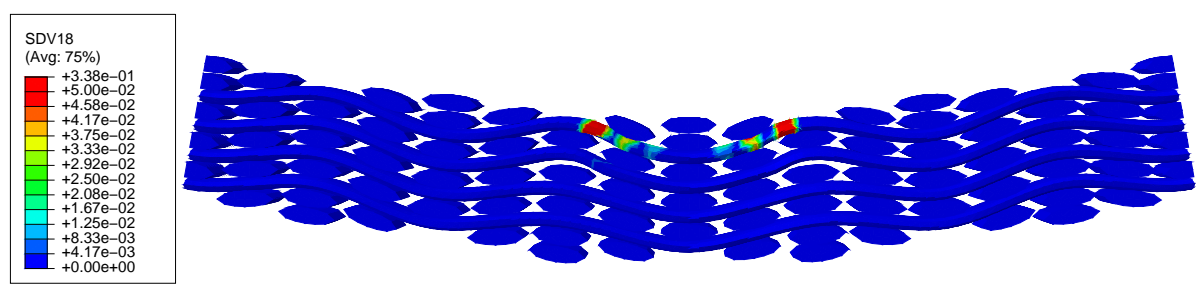

(c) At point (3)

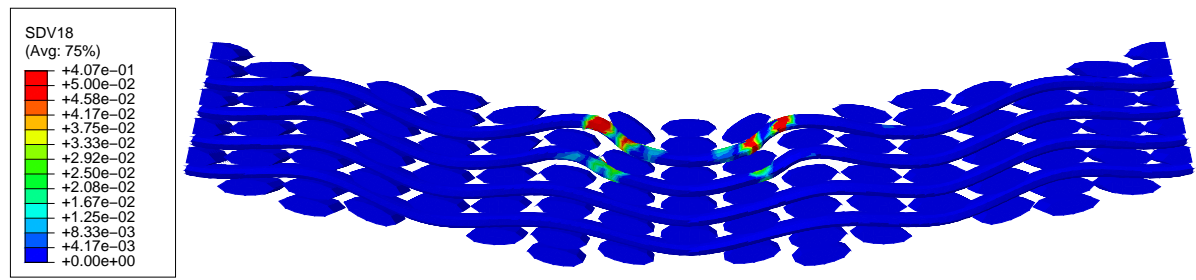

(d) At point (4)

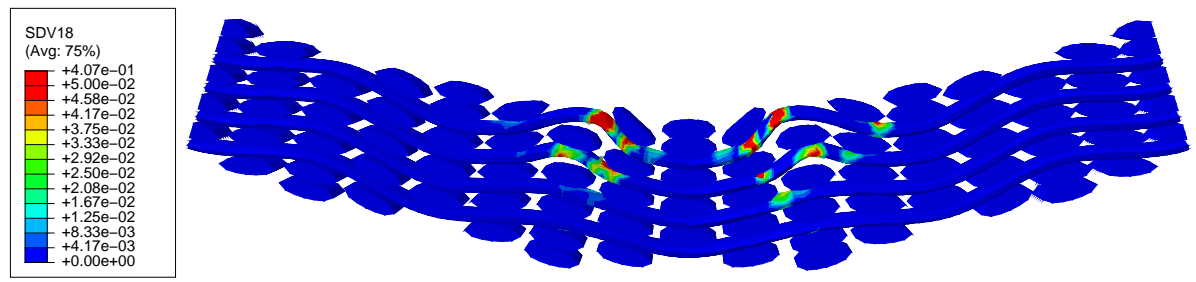

(e) At point (5)

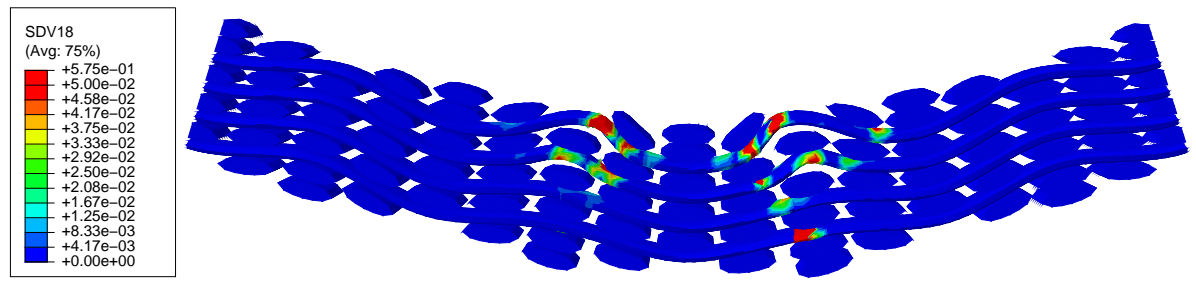

(f) At point (6)

Figure 20. Evolution of fiber tow crack strains during the deformation. The crack strains are shown in absolute values. 


\section{Appendix}

The five effective fiber tow constants defined in Section VI are computed based upon the fiber and matrix properties as,

$$
\begin{aligned}
E_{1}^{t} & =E_{1}^{f} V_{f}+E^{m}\left(1-V_{f}\right)+\frac{4 V_{f}\left(1-V_{f}\right)\left(\nu_{12}^{f}-\nu^{m}\right)^{2} G^{m}}{\frac{G^{m}\left(1-V_{f}\right)}{K_{23}^{f}}+\frac{G^{m} V_{f}}{K_{23}^{m}}+1} \\
\nu_{12}^{t} & =\nu_{12}^{f} V_{f}+\nu^{m}\left(1-V_{f}\right)+\frac{V_{f}\left(1-V_{f}\right)\left(\nu_{12}^{f}-\nu^{m}\right)\left(\frac{G^{m}}{K_{23}^{m}}-\frac{G^{m}}{K_{23}^{f}}\right)}{\frac{G^{m}\left(1-V_{f}\right)}{K_{23}^{f}}+\frac{G^{m} V_{f}}{K_{23}^{m}}+1} \\
G_{12}^{t} & =G^{m} \frac{G_{12}^{f}\left(1+V_{f}\right)+G^{m}\left(1-V_{f}\right)}{G_{12}^{f}\left(1-V_{f}\right)+G^{m}\left(1+V_{f}\right)} \\
K_{23}^{t} & =K_{23}^{m}+\frac{V_{f}}{\frac{1}{K_{23}^{f}-K_{23}^{m}}+\frac{1-V_{f}}{K_{23}^{m}+G^{m}}}
\end{aligned}
$$

$G_{23}^{t}$ is computed through a quadratic equation as,

$$
A\left(\frac{G_{23}^{c}}{G^{m}}\right)^{2}+B\left(\frac{G_{23}^{t}}{G^{m}}\right)+C=0
$$

where,

$$
\begin{aligned}
& A=a_{0}+a_{1} V_{f}+a_{2} V_{f}^{2}+a_{3} V_{f}^{3}+a_{4} V_{f}^{4} \\
& B=b_{0}+b_{1} V_{f}+b_{2} V_{f}^{2}+b_{3} V_{f}^{3}+b_{4} V_{f}^{4} \\
& C=c_{0}+c_{1} V_{f}+c_{2} V_{f}^{2}+c_{3} V_{f}^{3}+c_{4} V_{f}^{4}
\end{aligned}
$$

with,

$$
\begin{aligned}
& a_{0}=-2\left(G^{m}+K_{23}^{m}\right)\left[2 G_{23}^{f} G^{m}+K_{23}^{f}\left(G_{23}^{f}+G^{m}\right)\right]\left[2 G_{23}^{f} G^{m}+K_{23}^{m}\left(G_{23}^{f}+G^{m}\right)\right] \\
& a_{1}=8\left(G_{23}^{f}-G^{m}\right)\left[2 G_{23}^{f} G^{m}+K_{23}^{f}\left(G_{23}^{f}+G^{m}\right)\right]\left(G^{m^{2}}+G^{m} K_{23}^{m}+K_{23}^{m^{2}}\right) \\
& a_{2}=-12 K_{23}^{m^{2}}\left(G_{23}^{f}-G^{m}\right)\left[2 G_{23}^{f} G^{m}+K_{23}^{f}\left(G_{23}^{f}+G^{m}\right)\right] \\
& a_{3}=8\left\{G_{23}^{f^{2}} G^{m^{2}} K_{23}^{f}+G_{23}^{f^{2}} G^{m} K_{23}^{m}\left(K_{23}^{f}-G^{m}\right)+K_{23}^{m^{2}}\left[G_{23}^{f} G^{m}\left(G_{23}^{f}-2 G^{m}\right)+\right.\right. \\
& \left.\left.K_{23}^{f}\left(G_{23}^{f}-G^{m}\right)\left(G_{23}^{f}+G^{m}\right)\right]\right\} \\
& a_{4}=2\left(G_{23}^{f}-G^{m}\right)\left(2 G^{m}+K_{23}^{m}\right)\left[K_{23}^{f} G^{m} K_{23}^{m}-G_{23}^{f}\left(2 G^{m}\left(K_{23}^{f}-K_{23}^{m}\right)+K_{23}^{f} K_{23}^{m}\right)\right] \\
& b_{0}=4 G^{m}\left[2 G_{23}^{f} G^{m}+K_{23}^{f}\left(G_{23}^{f}+G^{m}\right)\right]\left[2 G_{23}^{f} G^{m}+K_{23}^{m}\left(G_{23}^{f}+G^{m}\right)\right] \\
& b_{1}=8 K_{23}^{m}\left(G_{23}^{f}-G^{m}\right)\left[2 G_{23}^{f} G^{m}+\left(G_{23}^{f}+G^{m}\right) K_{23}^{f}\right]\left(G^{m}-K_{23}^{m}\right) \\
& b_{2}=24 K_{23}^{m^{2}}\left(G_{23}^{f}-G^{m}\right)\left[2 G_{23}^{f} G^{m}+\left(G_{23}^{f}+G^{m}\right) K_{23}^{f}\right] \\
& b_{3}=-16\left\{G_{23}^{f^{2}} G^{m^{2}} K_{23}^{f}+G_{23}^{f^{2}} G^{m} K_{23}^{m}\left(K_{23}^{f}-G^{m}\right)+K_{23}^{m^{2}}\left[G_{23}^{f} G^{m}\left(G_{23}^{f}-2 G^{m}\right)+\right.\right. \\
& \left.\left.K_{23}^{f}\left(G_{23}^{f}-G^{m}\right)\left(G_{23}^{f}+G^{m}\right)\right]\right\} \\
& b_{4}=-4 G^{m}\left(G_{23}^{f}-G^{m}\right)\left[K_{23}^{f} G^{m} K_{23}^{m}-G_{23}^{f}\left(2 G^{m}\left(K_{23}^{f}-K_{23}^{m}\right)+K_{23}^{f} K_{23}^{m}\right)\right]
\end{aligned}
$$




$$
\begin{aligned}
c_{0} & =2 K_{23}^{m}\left[2 G_{23}^{f} G^{m}+K_{23}^{f}\left(G_{23}^{f}+G^{m}\right)\right]\left[2 G_{23}^{f} G^{m}+K_{23}^{m}\left(G_{23}^{f}+G^{m}\right)\right] \\
c_{1} & =8 K_{23}^{m^{2}}\left(G_{23}^{f}-G^{m}\right)\left[2 G_{23}^{f} G^{m}+K_{23}^{f}\left(G_{23}^{f}+G^{m}\right)\right] \\
c_{2} & =-12 K_{23}^{m^{2}}\left(G_{23}^{f}-G^{m}\right)\left[2 G_{23}^{f} G^{m}+K_{23}^{f}\left(G_{23}^{f}+G^{m}\right)\right] \\
c_{3} & =8\left\{G_{23}^{f^{2}} G^{m^{2}} K_{23}^{f}+G_{23}^{f^{2}} G^{m} K_{23}^{m}\left(K_{23}^{f}-G^{m}\right)+K_{23}^{m^{2}}\left[G_{23}^{f} G^{m}\left(G_{23}^{f}-2 G^{m}\right)+\right.\right. \\
& \left.\left.K_{23}^{f}\left(G_{23}^{f}-G^{m}\right)\left(G_{23}^{f}+G^{m}\right)\right]\right\} \\
c_{4} & =-2 K_{23}^{m}\left(G_{23}^{f}-G^{m}\right)\left[K_{23}^{f} G^{m} K_{23}^{m}-G_{23}^{f}\left(2 G^{m}\left(K_{23}^{f}-K_{23}^{m}\right)+K_{23}^{f} K_{23}^{m}\right)\right]
\end{aligned}
$$

The non-zero $F_{i j}$ components in Eq. (24) are computed using the CCM in conjunction with the GSCM, in which the unidirectional composite is represented by an inner fiber core and an outer matrix annulus. The derivation for the $\boldsymbol{F}$ matrix is provided in Ref., ${ }^{32}$ and the results are summarized in Eq. (35)-(39) as,

$$
\begin{aligned}
& F_{11}=1 \\
& F_{21}=\frac{V_{f}\left(\frac{\nu_{12}^{f}}{K_{23}^{m}}-\frac{\nu^{m}}{K_{23}^{f}}\right)}{\frac{V_{f}}{K_{23}^{f}}+\frac{1-2 \nu^{m}}{K_{23}^{f}}+\frac{1-V_{f}}{K_{23}^{m}}}\left[1+\left(\frac{b}{r}\right)^{2} \cos 2 \theta\right] \\
& F_{31}=\frac{V_{f}\left(\frac{\nu_{12}^{f}}{K_{23}^{m}}-\frac{\nu^{m}}{K_{23}^{f}}\right)}{\frac{V_{f}}{K_{23}^{f}}+\frac{1-2 \nu^{m}}{K_{23}^{f}}+\frac{1-V_{f}}{K_{23}^{m}}}\left[1-\left(\frac{b}{r}\right)^{2} \cos 2 \theta\right] \\
& F_{61}=2 \frac{V_{f}\left(\frac{\nu_{12}^{f}}{K_{23}^{m}}-\frac{\nu^{m}}{K_{23}^{f}}\right)}{\frac{V_{f}}{K_{23}^{f}}+\frac{1-2 \nu^{m}}{K_{23}^{f}}+\frac{1-V_{f}}{K_{23}^{m}}}\left(\frac{b}{r}\right)^{2} \sin 2 \theta \\
& F_{22}=\frac{1}{4 G^{m} \bar{\sigma}}\left\{\left[2 K_{23}^{t} \frac{G^{m}}{K_{23}^{m}} N_{2}-G_{23}^{t}\left(2 A_{2}+6 B_{2} \frac{r^{2}}{b^{2}}\right)\right]+\left[K_{23}^{t} M_{2} \frac{b^{2}}{r^{2}}\right.\right. \\
& \left.\left.+2 G_{23}^{t} \frac{G^{m}}{K_{23}^{m}}\left(3 B_{2} \frac{r^{2}}{b^{2}}-D_{2} \frac{b^{2}}{r^{2}}\right)\right] \cos 2 \theta-\left[G_{23}^{t}\left(3 C_{2} \frac{b^{4}}{r^{4}}+2 D_{2} \frac{b^{2}}{r^{2}}\right)\right] \cos 4 \theta\right\} \\
& F_{32}=\frac{1}{4 G^{m} \bar{\sigma}}\left\{\left[2 K_{23}^{t} \frac{G^{m}}{K_{23}^{m}} N_{2}+G_{23}^{t}\left(2 A_{2}+6 B_{2} \frac{r^{2}}{b^{2}}\right)\right]-\left[K_{23}^{t} M_{2} \frac{b^{2}}{r^{2}}\right.\right. \\
& \left.\left.-2 G_{23}^{t} \frac{G^{m}}{K_{23}^{m}}\left(3 B_{2} \frac{r^{2}}{b^{2}}-D_{2} \frac{b^{2}}{r^{2}}\right)\right] \cos 2 \theta+\left[G_{23}^{t}\left(3 C_{2} \frac{b^{4}}{r^{4}}+2 D_{2} \frac{b^{2}}{r^{2}}\right)\right] \cos 4 \theta\right\} \\
& F_{62}=\frac{1}{2 G^{m} \bar{\sigma}}\left[K_{23}^{t} M_{2} \frac{b^{2}}{r^{2}} \sin 2 \theta-G_{23}^{t}\left(3 C_{2} \frac{b^{4}}{r^{4}}+2 D_{2} \frac{b^{2}}{r^{2}}\right) \sin 4 \theta\right]
\end{aligned}
$$




$$
\begin{aligned}
F_{23}= & \frac{1}{4 G^{m} \bar{\sigma}}\left\{\left[2 K_{23}^{t} \frac{G^{m}}{K_{23}^{m}} N_{2}+G_{23}^{t}\left(2 A_{2}+6 B_{2} \frac{r^{2}}{b^{2}}\right)\right]+\left[K_{23}^{t} M_{2} \frac{b^{2}}{r^{2}}\right.\right. \\
& \left.\left.-2 G_{23}^{t} \frac{G^{m}}{K_{23}^{m}}\left(3 B_{2} \frac{r^{2}}{b^{2}}-D_{2} \frac{b^{2}}{r^{2}}\right)\right] \cos 2 \theta+\left[G_{23}^{t}\left(3 C_{2} \frac{b^{4}}{r^{4}}+2 D_{2} \frac{b^{2}}{r^{2}}\right)\right] \cos 4 \theta\right\} \\
F_{33}= & \frac{1}{4 G^{m} \bar{\sigma}}\left\{\left[2 K_{23}^{t} \frac{G^{m}}{K_{23}^{m}} N_{2}-G_{23}^{t}\left(2 A_{2}+6 B_{2} \frac{r^{2}}{b^{2}}\right)\right]-\left[K_{23}^{t} M_{2} \frac{b^{2}}{r^{2}}\right.\right. \\
& \left.\left.+2 G_{23}^{t} \frac{G^{m}}{K_{23}^{m}}\left(3 B_{2} \frac{r^{2}}{b^{2}}-D_{2} \frac{b^{2}}{r^{2}}\right)\right] \cos 2 \theta-\left[G_{23}^{t}\left(3 C_{2} \frac{b^{4}}{r^{4}}+2 D_{2} \frac{b^{2}}{r^{2}}\right)\right] \cos 4 \theta\right\} \\
F_{63}= & \frac{1}{2 G^{m} \bar{\sigma}}\left[K_{23}^{t} M_{2} \frac{b^{2}}{r^{2}} \sin 2 \theta+G_{23}^{t}\left(3 C_{2} \frac{b^{4}}{r^{4}}+2 D_{2} \frac{b^{2}}{r^{2}}\right) \sin 4 \theta\right] \\
F_{44}= & \frac{G_{12}^{f}+G^{m}}{G_{12}^{f}+G^{m}-V_{f}\left(G_{12}^{f}-G^{m}\right)}+\frac{V_{f}\left(G_{12}-G^{m}\right)}{G_{12}^{f}+G^{m}-V_{f}\left(G_{12}^{f}-G^{m}\right)}\left(\frac{b}{r}\right)^{2} \cos 2 \theta \\
F_{54}= & \frac{\left(G_{12}^{f}-G^{m}\right) V_{f}}{G_{12}^{f}+G^{m}-V_{f}\left(G_{12}^{f}-G^{m}\right)}\left(\frac{b}{r}\right)^{2} \sin 2 \theta \\
F_{26}= & \frac{G_{23}^{t}}{4 G^{m} \bar{\sigma}}\left[\left(3 C_{2} \frac{b^{4}}{r^{4}}+2 D_{2} \frac{b^{2}}{r^{2}}\right) \sin 4 \theta+2 \frac{G^{m}}{K_{23}^{m}}\left(3 B_{2} \frac{r^{2}}{b^{2}}-D_{2} \frac{b^{2}}{r^{2}}\right) \sin 2 \theta\right] \\
F_{36}= & \frac{G_{23}^{t}}{4 G^{m} \bar{\sigma}}\left[-\left(3 C_{2} \frac{b^{4}}{r^{4}}+2 D_{2} \frac{b^{2}}{r^{2}}\right) \sin 4 \theta+2 \frac{G^{m}}{K_{23}^{m}}\left(3 B_{2} \frac{r^{2}}{b^{2}}-D_{2} \frac{b^{2}}{r^{2}}\right) \sin 2 \theta\right] \\
F_{66}= & \frac{G_{23}^{t}}{2 G^{m} \bar{\sigma}}\left[-\left(2 A_{2}+6 B_{2} \frac{r^{2}}{b^{2}}\right)+\left(3 C_{2} \frac{b^{4}}{r^{4}}+2 D_{2} \frac{b^{2}}{r^{2}}\right) \cos 4 \theta\right]
\end{aligned}
$$

where $b$ is the matrix outer radius. The unknown constants, $N_{1}, M_{2}, N_{2}$, and $M_{3}$, are computed by solving Eq. (40).

$$
\left[\begin{array}{cccc}
1 & -\frac{1}{2}\left(\frac{1}{V_{f}}\right) & -1 & 0 \\
2 \frac{G_{23}^{f}}{K_{23}^{f}} & \frac{G_{23}^{f}}{G^{m}} \frac{1}{V_{f}} & -2 \frac{G_{23}^{f}}{K_{23}^{m}} & 0 \\
0 & \frac{1}{2} & 1 & -\frac{1}{2} \\
0 & -\frac{G_{23}}{G^{m}} & 2 \frac{G_{23}}{K_{23}^{m}} & 1
\end{array}\right]\left\{\begin{array}{l}
N_{1} \\
M_{2} \\
N_{2} \\
M_{3}
\end{array}\right\}=\left\{\begin{array}{c}
0 \\
0 \\
\bar{\sigma} \\
2 \frac{G_{23}}{K_{23}}
\end{array}\right\}
$$

The remaining unknown constants, $A_{1}, B_{1}, A_{2}, B_{2}, C_{2}, D_{2}, C_{3}$, and $D_{3}$ are determined from Eq. (41).

$$
[B]\{d\}=\{f\}
$$

where,

$$
B=\left[\begin{array}{cccccccc}
-1 & 0 & 1 & 0 & \frac{3}{2}\left(\frac{1}{V_{f}}\right)^{2} & 2 \frac{1}{V_{f}} & 0 & 0 \\
1 & 3 V_{f} & -1 & -3 V_{f} & \frac{3}{2}\left(\frac{1}{V_{f}}\right)^{2} & \frac{1}{V_{f}} & 0 & 0 \\
-2 & 2\left(\frac{G_{23}^{f}}{K_{23}^{f}}-1\right) V_{f} & 2 \frac{G_{23}^{f}}{G^{m}} & -2 \frac{G_{23}^{f}}{G^{m}}\left(\frac{G^{m}}{K_{23}^{m}}-1\right) V_{f} & -\frac{G_{23}^{f}}{G^{m}}\left(\frac{1}{V_{f}}\right)^{2} & -2 \frac{G_{23}^{f}}{G^{m}}\left(\frac{G^{m}}{K_{23}^{m}}+1\right) \frac{1}{V_{f}} & 0 & 0 \\
2 & 2\left(\frac{G_{23}^{f}}{K_{23}^{f}}+2\right) V_{f} & -2 \frac{G_{23}^{f}}{G^{m}} & -2 \frac{G_{23}^{f}}{G^{m}}\left(\frac{G^{m}}{K_{23}^{m}}+2\right) V_{f} & -\frac{G_{23}^{f}}{G^{m}}\left(\frac{1}{V_{f}}\right)^{2} & 2 \frac{G_{23}^{f}}{K_{23}^{m}} \frac{1}{V_{f}} & 0 & 0 \\
0 & 0 & -1 & 0 & -\frac{3}{2} & -2 & \frac{3}{2} & 2 \\
0 & 0 & 1 & 3 & -\frac{3}{2} & -1 & \frac{3}{2} & 1 \\
0 & 0 & -2 \frac{G_{23}}{G^{m}} & 2 \frac{G_{23}}{G^{m}}\left(\frac{G^{m}}{K_{23}^{m}}-1\right) & \frac{G_{23}}{G^{m}} & 2 \frac{G_{23}}{G^{m}}\left(\frac{G^{m}}{K_{23}^{m}}+1\right) & -1 & -2\left(\frac{G_{23}}{K_{23}}+1\right) \\
0 & 0 & 2 \frac{G_{23}}{G^{m}} & 2 \frac{G_{23}}{G^{m}}\left(\frac{G^{m}}{K_{23}^{m}}+2\right) & \frac{G_{23}}{G^{m}} & -2 \frac{G_{23}}{K_{23}} & -1 & 2 \frac{G_{23}}{K_{23}}
\end{array}\right]
$$




$$
\begin{aligned}
d & =\left[\begin{array}{lllllllll}
A_{1} & B_{1} & A_{2} & B_{2} & C_{2} & D_{2} & D_{2} & C_{3} & D_{3}
\end{array}\right]^{T} \\
f & =\left[\begin{array}{lllllllll}
0 & 0 & 0 & 0 & \bar{\sigma} & -\bar{\sigma} & 2 \bar{\sigma} & -2 \bar{\sigma}
\end{array}\right]^{T}
\end{aligned}
$$

\section{Acknowledgments}

The authors are grateful for the financial support from the Army Research Laboratory, Aberdeen Proving Ground, MD, and the Army Research Office. Dr. Larry Russell is the program monitor.

\section{References}

\footnotetext{
${ }^{1}$ Pankow, M., Salvi, A., Waas, A., Yen, C., and Ghiorse, S., "Resistance to delamination of 3D woven textile composites evaluated using End Notch Flexure (ENF) tests: Experimental results," Composites Part A: Applied Science and Manufacturing, Vol. 42, No. 10, Oct. 2011, pp. 1463-1476.

${ }^{2}$ Luo, Y., Lv, L., Sun, B., Qiu, Y., and Gu, B., "Transverse impact behavior and energy absorption of three-dimensional orthogonal hybrid woven composites," Composite Structures, Vol. 81, No. 2, Nov. 2007, pp. 202-209.

${ }^{3}$ Baucom, J. N. and Zikry, M. A., "Evolution of failure mechanisms in 2D and 3D woven composite systems under quasi-static perforation," Journal of Composite Materials, Vol. 37, 2003, pp. 1651-1674.

${ }^{4}$ Quek, S. C., Waas, A. M., Shahwan, K. W., and Agaram, V., "Analysis of 2D triaxial flat braided textile composites," International Journal of Mechanical Sciences, Vol. 45, No. 6-7, June 2003, pp. 1077-1096.

${ }^{5}$ Sankar, B. V. and Marrey, R. V., "Analytical method for micromechanics of textile composites," Composites Science and Technology, Vol. 57, No. 6, Jan. 1997, pp. 703-713.

${ }^{6}$ Pankow, M., Waas, A. M., Yen, C.-F., and Ghiorse, S., "A new lamination theory for layered textile composites that account for manufacturing induced effects," Composites Part A: Applied Science and Manufacturing, Vol. 40, No. 12, Dec. 2009, pp. 1991-2003.

${ }^{7}$ Cox, B., Dadkhah, M., Morris, W., and Flintoff, J., "Failure mechanisms of 3D woven composites in tension, compression, and bending," Acta Metallurgica et Materialia, Vol. 42, No. 12, Dec. 1994, pp. 3967-3984.

${ }^{8}$ Huang, H. J. and Waas, A. M., "Modeling and predicting the compression strength limiting mechanisms in Z-pinned textile composites," Composites Part B: Engineering, Vol. 40, No. 6, Sept. 2009, pp. 530-539.

${ }^{9}$ Rao, M., Sankar, B., and Subhash, G., "Effect of Z-yarns on the stiffness and strength of three-dimensional woven composites," Composites Part B: Engineering, Vol. 40, No. 6, Sept. 2009, pp. 540-551.

${ }^{10}$ Zhang, D., Pankow, M., Waas, A. M., Yen, C., and Ghiorse, S., "Flexural behavior of a layer-to-layer orthogonal interlocked three-dimensional textile composite," Journal of Engineering Materials and Technology, Vol. 134, 2012, pp. 031009$1-8$.

${ }^{11}$ Cox, B., Dadkhah, M., Inman, R., Morris, W., and Zupon, J., "Mechanisms of compressive failure in 3D composites," Acta Metallurgica et Materialia, Vol. 40, No. 12, Dec. 1992, pp. 3285-3298.

${ }^{12}$ Quek, S. C., Waas, A. M., Shahwan, K. W., and Agaram, V., "Failure mechanics of triaxially braided carbon composites under combined bendingcompression loading," Composites Science and Technology, Vol. 66, No. 14, Nov. 2006, pp. $2548-2556$.

${ }^{13}$ Budiansky, B. and Fleck, N., "Compressive failure of fibre composites," Journal of the Mechanics and Physics of Solids, Vol. 41, No. 1, Jan. 1993, pp. 183-211.

${ }^{14}$ Kyriakides, S., Arseculeratne, R., Perry, E. J., and Liechti, K. M., "On the compressive failure of fiber reinforced composites," International Journal of Solids and Structures, Vol. 32, No. 6/7, 1995, pp. 689-738.

${ }^{15}$ Lee, S. H. and Waas, A. M., "Compressive response and failure of fiber reinforced unidirectional composites," International Journal of Fracture, Vol. 100, No. 3, 1999, pp. 275-306.

${ }^{16}$ Feld, N., Allix, O., Baranger, E., and Guimard, J.-M., "Micro-mechanical prediction of UD laminates behavior under combined compression up to failure: influence of matrix degradation," Journal of Composite Materials, Vol. 45, No. 22, July 2011, pp. 2317-2333.

${ }^{17}$ Prabhakar, P. and Waas, A. M., "Upscaling from a micro-mechanics model to capture laminate compressive strength due to kink banding instability," Computational Materials Science, Vol. 67, Feb. 2013, pp. 40-47.

${ }^{18}$ Prabhakar, P. and Waas, A. M., "Interaction between kinking and splitting in the compressive failure of unidirectional fiber reinforced laminated composites," Composite Structures, Vol. 98, April 2013, pp. 85-92.

${ }^{19}$ Long, A. C., Design and manufacture of textile composites, Woodhead Publishing Limited in association with The Textile Institute, Cambridge, England, 1st ed., 2005.

${ }^{20}$ Pankow, M. R., The Deformation Response of 3D Woven Composites Subjected to High Rates of Loading, Ph.D. thesis, University of Michigan, 2010.

${ }^{21}$ Zhou, Y., Pervin, F., Biswas, M. a., Rangari, V. K., and Jeelani, S., "Fabrication and characterization of montmorillonite clay-filled SC-15 epoxy," Materials Letters, Vol. 60, No. 7, April 2006, pp. 869-873.

${ }^{22}$ Song, S., Waas, A. M., Shahwan, K. W., Xiao, X., and Faruque, O., "Braided textile composites under compressive loads: Modeling the response, strength and degradation," Composites Science and Technology, Vol. 67, No. 15-16, Dec. 2007, pp. 3059-3070.

${ }^{23}$ Sicking, D. L., Mechanical Characterization of Nonlinear Laminated Composites with Transverse Crack Growth, Ph.D. thesis, Texas A\&M University, 1992.
} 
${ }^{24} \mathrm{Ng}$, W. H., Salvi, A. G., and Waas, A. M., "Characterization of the in-situ non-linear shear response of laminated fiber-reinforced composites," Composites Science and Technology, Vol. 70, No. 7, July 2010, pp. 1126-1134.

${ }^{25}$ Rots, G., Nauta, P., Kusters, G. M. A., and Blaauwendraad, J., "Smeared crack approach and fracture localization in concrete," HERON, Vol. 30, No. 1, 1985, pp. 1-48.

${ }^{26}$ Heinrich, C. and Waas, A. M., "Investigation of progressive damage and fracture in laminated composites using the smeared crack approach," 53rd AIAA/ASME/ASCE/AHS/ASC Structures, Structural Dynamics and Materials Conference, Honolulu, Hawaii, April 2012, AIAA 2012-1537.

${ }^{27}$ Bažant, Z. P. and Gambarova, P. G., "Rough cracks in reinforced concrete," Journal of the Structural Division, Vol. 106, 1980, pp. 819-842.

${ }^{28}$ Walraven, J. C., Aggregate Interlock: A Theoretical and Experimental Analysis., Ph.D. thesis, Delft University of Technology, 1980.

${ }^{29}$ Walraven, J. C. and Reinhardt, H. W., "Theory and experiements on the mechanical behaviour of cracks in plain and reinforced concrete subjected to shear loading," HERON, Vol. 26, No. 1A.

${ }^{30}$ Gambarova, P. G. and Karakoç, C., "A new approach to the analysis of the confinement role in regularly cracked concrete elements," Transactions 7th SMIRT Conference H (Paper H5/7)(Chicago, 1983), 1983, pp. 251-261.

${ }^{31}$ Camanho, P. P., Dávila, C. G., Pinho, S. T., Iannucci, L., and Robinson, P., "Prediction of in situ strengths and matrix cracking in composites under transverse tension and in-plane shear," Composites Part A: Applied Science and Manufacturing, Vol. 37, No. 2, Feb. 2006, pp. 165-176.

${ }^{32}$ Zhang, D. and Waas, A. M., "A micromechanics based multiscale model for nonlinear composites," Acta Mechanica, submitted for publication.

${ }^{33}$ Hashin, Z. and Rosen, B. W., "The elastic moduli of fiber-reinforced materials," Journal of Applied Mechanics, Vol. 31, No. 2, 1964, pp. 223-232.

${ }^{34}$ Christensen, R. and Lo, K., "Solutions for effective shear properties in three phase sphere and cylinder models," Journal of the Mechanics and Physics of Solids, Vol. 27, No. 4, Aug. 1979, pp. 315-330.

${ }^{35}$ Pankow, M., Salvi, A., Waas, A., Yen, C., and Ghiorse, S., "Split Hopkinson pressure bar testing of 3D woven composites," Composites Science and Technology, Vol. 71, No. 9, June 2011, pp. 1196-1208.

${ }^{36}$ Schultheisz, C. R. and Waas, A. M., "Compressive failure of composites, part I: Testing and micromechanical theories," Progress in Aerospace Sciences, Vol. 32, No. 1, Jan. 1996, pp. 1-42.

${ }^{37}$ Waas, A. M. and Schultheisz, C. R., "Compressive failure of composites, part II: Experimental studies," Progress in Aerospace Sciences, Vol. 32, No. 1, Jan. 1996, pp. 43-78.

${ }^{38}$ Basu, S., Waas, A. M., and Ambur, D. R., "A macroscopic model for kink banding instabilites in fiber composites," Journal of Mechanics of Materials and Structures, Vol. 1, No. 6, 2006.

${ }^{39}$ Feld, N., Allix, O., Baranger, E., and Guimard, J.-M., "A micromechanics-based mesomodel for unidirectional laminates in compression up to failure," Journal of Composite Materials, Vol. 46, No. 23, Jan. 2012, pp. 2893-2909.

${ }^{40}$ Vogler, T., Hsu, S.-Y., and Kyriakides, S., "On the initiation and growth of kink bands in fiber composites. Part II: analysis," International Journal of Solids and Structures, Vol. 38, No. 15, April 2001, pp. 2653-2682.

${ }^{41}$ Hsu, S.-Y., Vogler, T., and Kyriakides, S., "On the axial propagation of kink bands in fiber composites : Part II analysis," International Journal of Solids and Structures, Vol. 36, No. 4, Feb. 1999, pp. 575-595.

${ }^{42}$ Yerramalli, C. S. and Waas, A. M., "The effect of fiber diameter on the compressive strength of composites - A 3D finite element based study," Computer Modeling in Engineering and Sciences, Vol. 6, No. 1, 2004, pp. 1-16.

${ }^{43}$ Schapery, R., "A theory of mechanical behavior of elastic media with growing damage and other changes in structure," Journal of the Mechanics and Physics of Solids, Vol. 38, No. 2, Jan. 1990, pp. 215-253.

${ }^{44}$ Hill, R., "A theory of the yielding and plastic flow of anisotropic metals," Proceedings of the Royal Society A: Mathematical, Physical and Engineering Sciences, Vol. 193, No. 1033, May 1948, pp. 281-297.

${ }^{45}$ Song, S., Waas, A. M., Shahwan, K. W., Faruque, O., and Xiao, X. S., "Compression response, strength and post-peak response of an axial fiber reinforced tow," International Journal of Mechanical Sciences, Vol. 51, No. 7, July 2009, pp. 491-499.

${ }^{46}$ Totry, E., González, C., and LLorca, J., "Influence of the loading path on the strength of fiber-reinforced composites subjected to transverse compression and shear," International Journal of Solids and Structures, Vol. 45, No. 6, March 2008, pp. $1663-1675$.

${ }^{47}$ Totry, E., González, C., and LLorca, J., "Prediction of the failure locus of C/PEEK composites under transverse compression and longitudinal shear through computational micromechanics," Composites Science and Technology, Vol. 68, No. 15-16, Dec. 2008, pp. 3128-3136.

${ }^{48}$ Totry, E., Molina-Aldareguía, J. M., González, C., and LLorca, J., "Effect of fiber, matrix and interface properties on the in-plane shear deformation of carbon-fiber reinforced composites," Composites Science and Technology, Vol. 70, No. 6, June 2010, pp. 970-980.

${ }^{49}$ Pineda, E. J., Bednarcyk, B. A., Waas, A. M., and Arnold, S. M., "Progressive failure of a unidirectional fiber-reinforced composite using the method of cells: Discretization objective computational results," International Journal of Solids and Structures, Vol. 50, No. 9, May 2013, pp. 1203-1216.

${ }^{50}$ Hashin, Z., "Failure Criteria for Unidirectional Fiber Composites," Journal of Applied Mechanics, Vol. 47, No. 2, 1980, pp. 329 .

${ }^{51}$ Puck, A., "Failure analysis of FRP laminates by means of physically based phenomenological models," Composites Science and Technology, Vol. 58, No. 7, July 1998, pp. 1045-1067.

${ }^{52}$ Puck, A. and Schürmann, H., "Failure analysis of FRP laminates by means of physically based phenomenological models," Composites Science and Technology, Vol. 62, No. 12-13, Sept. 2002, pp. 1633-1662.

${ }^{53}$ Camanho, P., Maimí, P., and Dávila, C., "Prediction of size effects in notched laminates using continuum damage mechanics," Composites Science and Technology, Vol. 67, No. 13, Oct. 2007, pp. 2715-2727. 
${ }^{54}$ Laffan, M., Pinho, S., Robinson, P., Iannucci, L., and McMillan, A., "Measurement of the fracture toughness associated with the longitudinal fibre compressive failure mode of laminated composites," Composites Part A: Applied Science and Manufacturing, Vol. 43, No. 11, Nov. 2012, pp. 1930-1938.

${ }^{55}$ AGY, "High Strength Glass Fibers," 2006, http://www.agy.com/technical_info/graphics_PDFs/ HighStrengthTechPaperEng.pdf. 